\title{
Geochemistry of Cretaceous Mudrocks and Sandstones from Douala Sub-Basin, Kumba Area, South West Cameroon: Constraints on Provenance, Source Rock Weathering, Paleo-Oxidation Conditions and Tectonic Environment
}

\author{
${ }^{1}$ Department of Earth Sciences, University of Yaoundé 1, Yaoundé, Cameroon \\ ${ }^{2}$ Department of Earth Sciences, University of Maroua, Maroua, Cameroon \\ ${ }^{3}$ Department of Earth Sciences, University of Dschang, Dschang, Cameroon \\ Email: *ngueutchoua2@yahoo.fr
}

Gabriel Ngueutchoua ${ }^{1}$, Leopold Darlus Ngantchu ${ }^{1}$, Martine Youbi ${ }^{1}$, Simon Ngos III ${ }^{2}$, Véronique Kamgang Kabeyene Beyala ${ }^{3}$, Kevin Petsouonko Yifomjuํㅜ, Jean Claude Tchamgoué ${ }^{1}$

How to cite this paper: Ngueutchoua, G., Ngantchu, L.D., Youbi, M., Ngos III, S., Beyala, V.K.K., Yifomju, K.P. and Tchamgoué, J.C. (2017) Geochemistry of Cretaceous Mudrocks and Sandstones from Douala Sub-Basin, Kumba Area, South West Cameroon: Constraints on Provenance, Source Rock Weathering, Paleo-Oxidation Conditions and Tectonic Environment. International Journal of Geosciences, 8, 393-424. https://doi.org/10.4236/ijg.2017.84021

Received: March 1, 2017

Accepted: April 14, 2017

Published: April 17, 2017

Copyright ( 92017 by authors and Scientific Research Publishing Inc. This work is licensed under the Creative Commons Attribution International License (CC BY 4.0).

http://creativecommons.org/licenses/by/4.0/ (c) (i) Open Access

\begin{abstract}
Geochemical data of fifteen Cretaceous sediment samples from Kumba area in the Douala sub-basin are presented to determine the provenance, source rock weathering, tectonic setting and paleo-oxidation conditions of the depositional setting of these rocks. For this purpose, the whole-rocks were analyzed for their major and trace element, including rare earth elements (REEs), contents by ICP-AES and ICP-MS methods respectively. On the basis of their major element composition, the rocks have been classified mainly as Fe-shale, shale, arkose and $\mathrm{Fe}$-sandstone. For the provenance, the plot of $\mathrm{Zr}$ vs. $\mathrm{TiO}_{2}, \mathrm{Y} / \mathrm{Ni}$ vs. $\mathrm{Cr} / \mathrm{V}, \mathrm{TiO}_{2}$ vs. $\mathrm{Al}_{2} \mathrm{O}_{3}$ diagrams, high LREE/HREE ratios (5.84 to 20.91) and negative and positive $\mathrm{Eu}$ anomalies $\left(\mathrm{Eu} / \mathrm{Eu}^{*}=0.87\right.$ to 1.62$)$ suggest that the studied rocks were mainly derived from felsic igneous rocks with lesser contribution of mafic components. The higher values of paleo-weathering indices such as Plagioclase Index of Alteration (PIA) and Chemical Index of Weathering (CIW), ranging from 79.63 to 99.90 and 87.57 to 99.92 respectively, suggest that the sediments and their potential source rocks experienced intense weathering. Whereas the variable Chemical Index of Alteration (CIA) values (63.01 to 99.50) coupled with high content of $\mathrm{K}_{2} \mathrm{O}$ (up to 7.5) in most samples could be indicative of $\mathrm{K}$-addition diagenetic processes. The A-CN-K plot also suggests a possible $\mathrm{K}$-addition. The redox conditions during the sedimentation were suboxic to oxic as evidenced by the Ce anomalies of 0.88 to
\end{abstract}


1.03. This is also confirmed by the values of $\mathrm{Ni} / \mathrm{Co}$ (less than 7), U/Th (less than 1.25) and $\mathrm{Cu} / \mathrm{Zn}$ (mostly less than 1). Tectonic discrimination diagrams (e.g., $\mathrm{SiO}_{2}-\mathrm{K}_{2} \mathrm{O} / \mathrm{Na}_{2} \mathrm{O}$ and $\mathrm{Th}-\mathrm{Sc}-\mathrm{Zr} / 10$ ) show that these rocks were deposited mainly in an active continental margin setting, and in various tectonic environments. This reflects probably the recycling effect experienced by the samples studied.

\section{Keywords}

Douala Sub-Basin, Provenance, Weathering, Oxic Condition, Tectonic Setting

\section{Introduction}

The Douala/Kribi-Campo basin is one of a series of continental shelf basins extending in West Africa from the edge of the Niger delta in Cameroon to the Walvis ridge near the Angola-Namibia border. It is divided into two sub-basins: the Kribi-Campo sub-basin to the south and the Douala sub-basin to the north. The latter is located at the bottom of the gulf of Guinea in the Central African rain forest. It comprises the Early to Mid-Cretaceous siliciclastic rocks such as sandstone, siltstone and shale. These deposits are known mainly from offshore boreholes because they have been interesting for petroleum industry [1]. As a result many geological studies (stratigraphy, sedimentology, etc.) were made in order to constraint the productive petroleum systems [1]-[7]. The onshore outcrops of these clastic sediments are not easily visible due to the dense landscape and the thick lateritic cover in the rainforest. Few areas such as Kumba exhibit these Lower to Mid Cretaceous outcrops which have been less described in the framework of a regional geology [8]. Until now, no geochemical studies have been carried out on the outcrops of ancient sedimentary rocks of Douala sub-basin exposed in the Kumba area.

The chemical composition of sedimentary rocks has been widely used to record the geological evolution of the continental crust through time [9]. Particularly, the geochemical signature, comprising of major, trace and rare earth elements (REEs) geochemistry, of siliciclastic sediments is indicative of several interacting variables such as provenance, weathering, transportation and diagenesis [10] [11] [12] [13]. A number of recent geochemical studies on ancient and modern clastic sediments from different parts of the world have been carried out and provided some valuable information on the source rock composition [14][20], climate, mode of transportation, depositional environment, degree of source rock weathering [21] [22] [23], tectonic setting and post depositional processes such as diagenesis and metasomatism [24] [25] [26] [27] [28].

In this study, inorganic geochemistry (major, trace and rare earth element composition) of Cretaceous mudrocks (siltstone and shale) and sandstones of Douala sub-basin exposed in the Kumba area in the South West Cameroon is presented to decipher provenance, degree of paleo-weathering, post-depositional diagenesis, paleo-oxidation conditions and tectonic setting of depositional envi- 
ronment. Considering the lack of geochemical data from the studied rocks, the data and their interpretation presented in this study will improve the understanding of the paleogeographical knowledge of the Douala sub-basin.

\section{Geological Setting}

The Douala sub-basin is located $3^{\circ} 20^{\prime} \mathrm{N}$ to $5^{\circ} \mathrm{N}$ and $9^{\circ} \mathrm{E}$ to $10^{\circ} 30^{\prime} \mathrm{E}$ (Figure 1). It occupies an emergent area of about $6950 \mathrm{~km}^{2}$. This sub-basin is limited to the South by the northern border of the Kribi-Campo sub-basin, which is broadly delineated by the northern end of the Kribi Fracture Zone (KFZ) [32] [33] (Figure 1 ), and to the Northwest and West by the Cameroon Volcanic Line (CVL), which also separates this sub-basin from the Rio-Del-Rey basin. Eastward, the boundary is the Pan-African migmatitic-gneiss basement, which is cross-cut in many places by mafic dykes and intruded by mafic to felsic plutons such as those of Ngongo and Ekomédion area [34] [35] (Figure 1(a) \& Figure 1(b)). The Douala sub-basin is semicircular in shape. The history of this sub-basin, like that of the of the Douala/Kribi-Campo basin to which it belongs, is related to the continental rifting, in the Late Jurassic, and the progressively separation of South American and African plates to form the South Atlantic Ocean [32] [36]. Its deposition comprises several formations which can be divided into three main tectono-lithostratigraphic sequences (corresponding to rift, rift-drift and drift stage) ranging in age from Early Cretaceous to Pleistocene, according to previous studies [3] [33] [36] [37] (Figure 2). Among these, Cretaceous sequences shown consist of four lithostratigraphic formations, namely, from bottom to top, the Lower Mundeck, Upper Mundeck, Logbadjeck and Logbaba (Figure 2).

The Lower Mundeck Formation formerly named "Basal sandstones" (Barremian-Aptian) represents the oldest deposit (syn-rift sequence) known from Douala sub-basin [1] [3] [37] [38] [39]. It consists of conglomerates, conglomeratic sandstones, arkosic sandstones, organic matter-rich dark marls interbedded with thin limestones and black to dark grey shales. This synrift sequence unconformably overlies Precambrian basement and appears to be controlled by listric faulting and associate roll-over anticlines [36]. The Lower Mundeck Formation is theoretically overlain by a relatively thin transitional evaporate unit. The latter has been observed in the neighboring Kribi-Campo sub-basin, but has not yet been recognized in the Douala sub-basin. This salt deposition corresponds to the rift-drift transition phase (Mid-Late Aptian). This rift-drift sequence was marked by transform directions resulting in a series of cross-faults which have segmented the rift structure [40].

The Upper Mundeck Formation (Albian-Cenomanian) consisting of sandstones, shales, marls and carbonates was deposited under marine conditions. These shales and sandstones are exposed at the Kumba area (Figure 3). The Upper Mundeck Formation unconformably overlies the Lower Mundeck formation and corresponds to the first drift stage (Albian-Coniacian, noted Drift I; Figure 2) of the post rift phase (Albian-Present).

The Logbadjeck Formation (Cenomanian-Campanian) is represented by an 


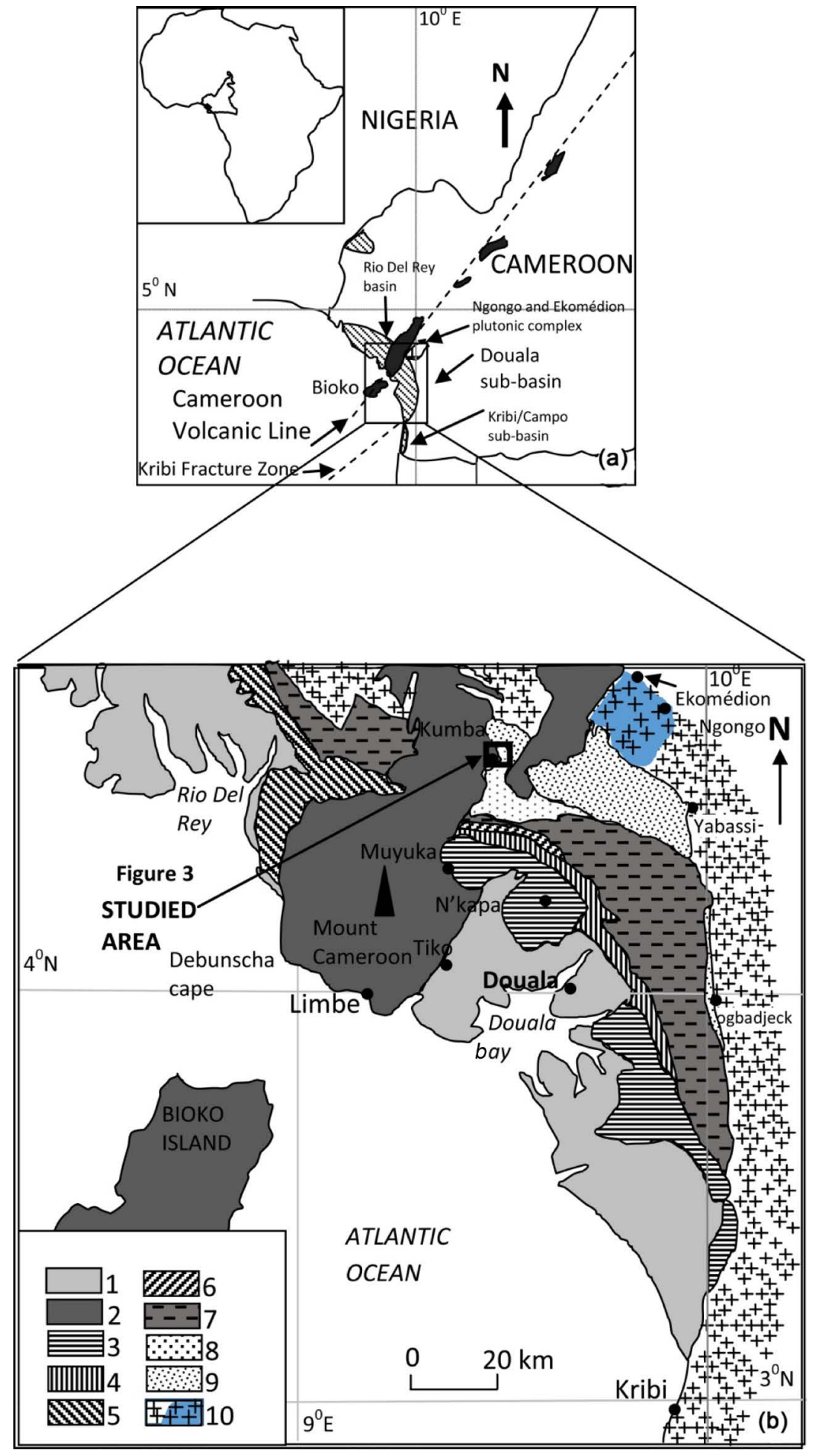

Figure 1. (a) Location of the study site and (b) geological map of this site and Kumba area (geological map adapted from [29] [30] [31]: (1) Recent alluvium, (2) Tertiary volcanic rocks (basalts and trachytes), (3) Neogene (siltstones, sandstones), (4) Lower Eocene (bedded clays, claystones, silts, loose sandstones), (5) Undifferenciated Tertiary, (6) Paleocene (marine facies: claystones, dolomites, sandstones, silts), (7) Paleocene (continental facies: small conglomerates, loose sandstones), (8) Upper Cretaceous (clays, sands, sandstones, marly and calcareous limestones), (9) Lower Cretaceous (Basalt sandstone), (10) Precambrian basement (migmatitic gneisses + granites). 


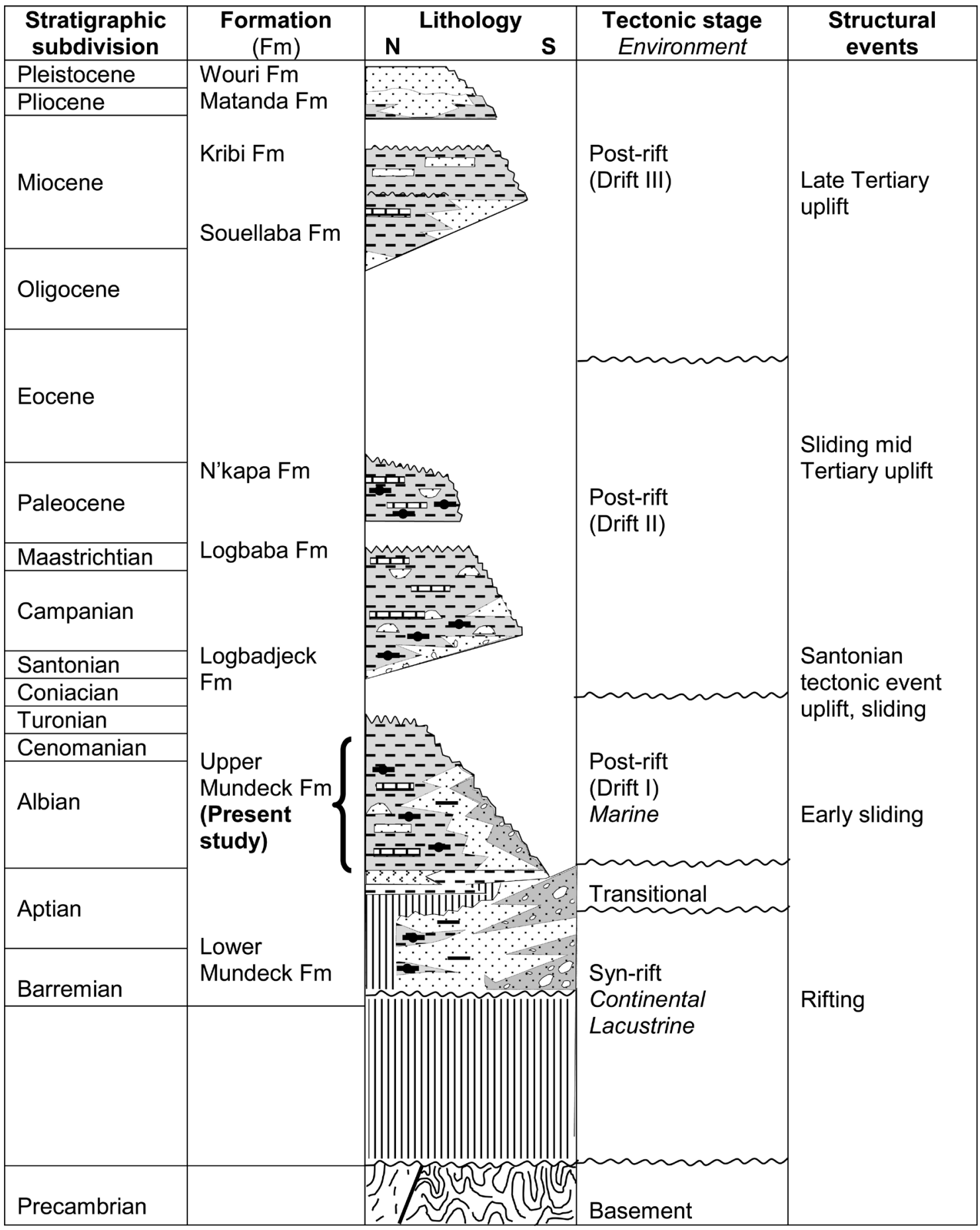

P) Metamorphic rocks

$0 \quad$ Conglomerates

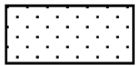

푸료
Sandstones

Clayey sandstones

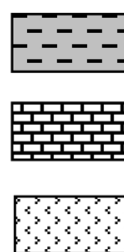

Claystones

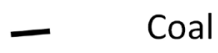

Carbonates $\sim$ Organic rich shales

Evaporite Unconformity

Hiatus

Figure 2. Tectono-lithostratigraphy of Douala sub-basin. Compiled from [3] [33] and [36]. 


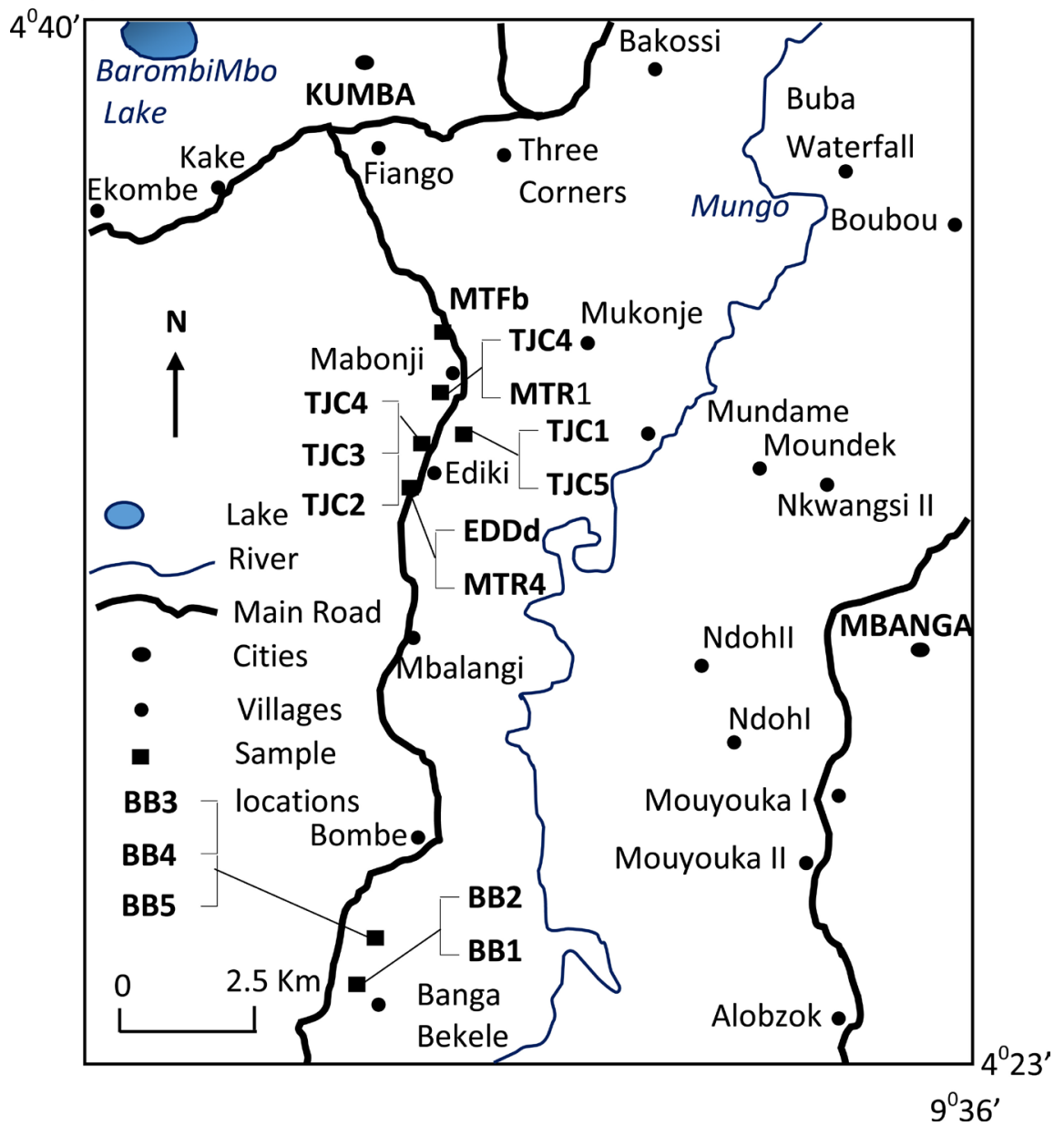

Figure 3. Sample locations (black squares) of the Kumba sediments. Insets show the stratigraphic position of samples from vertical section. For the map location, see Figure 1.

assemblage that is mainly clayey, with sandy intercalations and rare episodes of foraminifera-rich limestone [8]. This formation represents the first deposit of the second drift stage (Santonian-Eocene, noted Drift II; Figure 2). It is a discrete drift phase linked to a regional tectonic episode. During this episode, original rift-related roll-over structures were inverted and the platform sedimentary section was folded. The Drift II is separated from the Drift I by the Santonian unconformity.

The Logbaba Formation (Maastrichtian) is constituted of shales with calcareous nodules. It is dated due to its pollens and its rich microfauna [8]. This formation also belongs to the second drift stage.

This study is focused on clastic rocks belonging from the Upper Mundeck Formation (Albian-Cenomanian), outcropping at the Kumba area.

\section{Sampling and Analytical Methodology}

After a careful geological mapping, a total of fifteen representative sediment samples were collected from Lower to Mid Cretaceous succession from Douala sub-basin, exposed at Kumba area. These samples, consisting of five shales, four 
siltstones (both termed as mudrocks) and six sandstones, were trimmed to remove weathered surfaces and subsequently crushed using a jaw crusher for size reduction. The particles were oven-dried at $110^{\circ} \mathrm{C}$ overnight. The dried samples were then left to cool for $24 \mathrm{~h}$. The samples were pulverized in an agate mortar to grain size of less than $0.063 \mathrm{~mm}$, homogenized and packed into plastic bags. Five grams of aliquots of each powdered sample were packed and sent to ALS Geochemical Laboratory, Galway, Ireland for major and trace elements (including rare earth elements-REEs) determination.

Major and trace elements (including REEs) were determined by Inductively Coupled Plasma-Atomic Emission Spectrometry (ICP-AES) and Inductively Coupled Plasma Mass Spectrometer (ICP-MS), respectively. For ICP analyses, $0.25 \mathrm{~g}$ aliquots of each sample were mixed with a flux of lithium metaborate and lithium tetra borate and fused in an induction furnace at $1000^{\circ} \mathrm{C}$. The molten melt was immediately poured into a solution of dilute $\mathrm{HNO}_{3}$. The digested samples were measured for major oxides and the trace elements. The oxides of 10 major elements ( $\mathrm{Si}, \mathrm{Ti}, \mathrm{Al}, \mathrm{Fe}, \mathrm{Mn}, \mathrm{Mg}, \mathrm{Ca}, \mathrm{Na}, \mathrm{K}$ and $\mathrm{P}$ ) were measured after the method described by [41]. Trace elements data (including REE) were obtained with standard ICP-MS procedure as described in detail by [42]. Detection limit were $0.01 \mathrm{wt} \%$ for all major elements. The precision of the analysis is within 0.01 - 2 ppm for most trace elements (except for $\mathrm{Li}, \mathrm{Ti}, \mathrm{Cr}$ and As which is less than $10 \mathrm{ppm}$ ). Loss on ignition (LOI) was determined from the weight loss after roasting the sample at $1000^{\circ} \mathrm{C}$ for 2 hours. Totals ranged between $98.28 \mathrm{wt} \%$ and $101.96 \mathrm{wt} \%$ for major elements. Further details of the analytical procedures, precision, accuracy and standards used for instrumental calibration can be obtained from the ALS Laboratories. The REEs data were compared to the average compositions of plutonic rocks intruding the Pan-African migmatitic-gneiss basement such as mafic to felsic intrusive rocks (MFR), fine-grained granites (FGG) and coarse-grained granites (CGG) [34], and biotite-rich granite, porphyritic granite, alkali feldspar granite and two-mica granite [35] which could be the potential source-rocks of Kumba Cretaceous sediments.

\section{Results}

\subsection{Major Elements}

The mudrock and sandstone samples from Kumba area show variable geochemical composition. These geochemical characteristics are plotted on the $\mathrm{SiO}_{2} /$ $\mathrm{Al}_{2} \mathrm{O}_{3}$ vs. $\mathrm{Fe}_{2} \mathrm{O} / \mathrm{K}_{2} \mathrm{O}$ discriminant diagram of Herron [43]. This scheme of classification has been proven to be useful when applied to clastic sedimentary rock [44]. Mudrocks are divided into Fe-shale, shale and wacke. The latter have been grouped into shale because of the less number of sample in each sub-group and due to the fact that the wacke samples plot toward the field of shale, thus reflecting variation in quartz-feldpar/mica ratio in the studied mudrocks. Sandstones are divided into arkose and Fe-sandstone (Figure 4). Table 1 shows the concentrations of major element oxide, different elemental ratios and presents the descriptions of different geochemical indices calculated for the sample. 


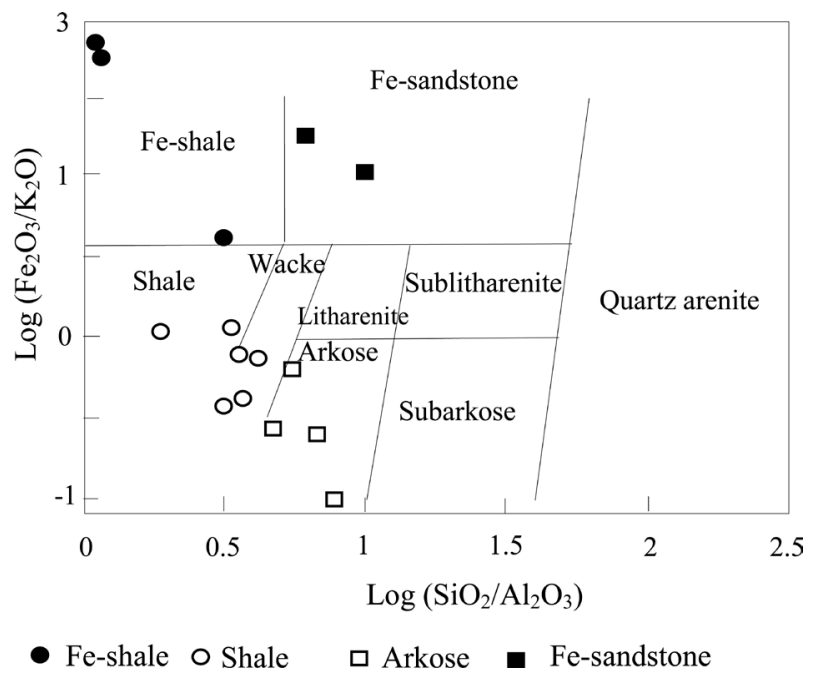

Figure 4. Classification of the Kumba sediments according to the scheme of Herron [43].

Table 1. Major elements composition (wt.\%) and element ratios of Kumba Cretaceous sediments and average compositions of PAAS and UCC.

\begin{tabular}{|c|c|c|c|c|c|c|c|c|c|c|c|c|c|c|c|c|c|}
\hline \multirow{2}{*}{$\begin{array}{c}\text { Sample } \\
\text { Ref. code }\end{array}$} & \multicolumn{3}{|c|}{$\underline{\text { Fe-shale }}$} & \multicolumn{6}{|c|}{$\underline{\text { Shale }}$} & \multicolumn{4}{|c|}{$\underline{\text { Arkose }}$} & \multicolumn{2}{|c|}{$\underline{\text { Fe-sandstone }}$} & \multirow[b]{2}{*}{ PAAS } & \multirow[b]{2}{*}{ UCC } \\
\hline & BB2 & BB3 & BB4 & EDDd & MTR5 & MTR4 & TJC1 & TJC2 & TJC4 & $\mathrm{MTFb}$ & MTR1 & TJC3 & TJC5 & BB5 & BB1 & & \\
\hline $\mathrm{SiO}_{2}$ & 30.40 & 24.60 & 59.40 & 65.10 & 62.20 & 55.00 & 56.90 & 61.50 & 62.10 & 70.80 & 84.10 & 67.30 & 77.80 & 79.80 & 76.80 & 62.80 & 66.00 \\
\hline $\mathrm{TiO}_{2}$ & 5.39 & 4.49 & 1.35 & 0.30 & 0.91 & 0.94 & 0.96 & 0.75 & 1.21 & 0.62 & 0.12 & 0.39 & .19 & 1.07 & 1.07 & 1.00 & 0.50 \\
\hline $\mathrm{Al}_{2} \mathrm{O}_{3}$ & 25.60 & 21.90 & 18.60 & 16.55 & 17.15 & 24.20 & 16.60 & 14.90 & 19.40 & 15.10 & 11.00 & 13.10 & 11.70 & 8.03 & 13.05 & 18.98 & 15.20 \\
\hline $\mathrm{Fe}_{2} \mathrm{O}_{3}$ & 22.60 & 33.20 & 4.39 & 2.45 & 5.72 & 4.33 & 7.52 & 3.56 & 2.49 & 1.75 & 0.17 & 2.61 & 0.97 & 6.22 & 3.54 & 6.50 & 4.50 \\
\hline $\mathrm{MnO}$ & 0.11 & 0.08 & 0.02 & 0.01 & 0.02 & 0.02 & 0.01 & 0.09 & 0.01 & 0.01 & 0.04 & 0.07 & 0.01 & 0.02 & 0.01 & 0.11 & 0.10 \\
\hline $\mathrm{MgO}$ & 0.37 & 0.19 & 0.42 & 0.22 & 1.46 & 1.20 & 1.58 & 1.87 & 0.93 & 0.81 & 0.06 & 1.24 & 0.10 & 0.06 & 0.09 & 2.20 & 2.20 \\
\hline $\mathrm{CaO}$ & 0.12 & 0.02 & 0.06 & 0.11 & 0.31 & 0.01 & 0.39 & 1.71 & 0.04 & 0.23 & $<0.01$ & 1.72 & 0.02 & $<0.01$ & 0.03 & 1.30 & 4.20 \\
\hline $\mathrm{Na}_{2} \mathrm{O}$ & 0.08 & 0.04 & 0.11 & 1.35 & 0.11 & 0.01 & 0.10 & 0.11 & 0.11 & 0.13 & 0.05 & 0.14 & 0.14 & 0.06 & 0.03 & 1.20 & 3.90 \\
\hline $\mathrm{K}_{2} \mathrm{O}$ & 0.08 & 0.05 & 1.13 & 6.36 & 7.50 & 4.03 & 7.01 & 6.45 & 6.60 & 7.19 & 2.11 & 5.83 & 4.39 & 0.63 & 0.13 & 3.70 & 3.40 \\
\hline $\mathrm{P}_{2} \mathrm{O}_{5}$ & 0.66 & 0.53 & 0.11 & 0.06 & 0.12 & 0.07 & 0.15 & 0.13 & 0.21 & 0.10 & 0.02 & 0.09 & 0.05 & 0.12 & 0.12 & 0.16 & 0.17 \\
\hline LOI & 13.60 & 13.45 & 13.55 & 8.87 & 6.14 & 11.15 & 7.15 & 7.77 & 6.39 & 4.37 & 3.40 & 7.01 & 2.91 & 4.51 & 6.09 & - & - \\
\hline Total & 99.01 & 98.55 & 99.14 & 101.38 & 101.64 & 101.96 & 98.37 & 98.84 & 99.49 & 101.11 & 101.07 & 99.50 & 98.28 & 100.52 & 100.96 & 97.77 & 100.10 \\
\hline $\begin{array}{l}\mathrm{SiO}_{2} / \\
\mathrm{Al}_{2} \mathrm{O}_{3}\end{array}$ & 1.19 & 1.20 & 3.90 & 3.93 & 3.63 & 2.27 & 3.42 & 4.12 & 3.20 & 4.69 & 7.65 & 5.13 & 6.64 & 9.94 & 5.89 & 3.32 & 4.34 \\
\hline $\begin{array}{l}\mathrm{K}_{2} \mathrm{O} / \\
\mathrm{Na}_{2} \mathrm{O}\end{array}$ & 1.00 & 1.25 & 10.27 & 4.71 & 68.18 & 403.00 & 70.10 & 58.63 & 60.00 & 55.31 & 42.20 & 41.64 & 31.35 & 10.50 & 4.33 & 3.08 & 0.87 \\
\hline $\begin{array}{l}\mathrm{K}_{2} \mathrm{O} / \\
\mathrm{Al}_{2} \mathrm{O}_{3}\end{array}$ & 0.00 & 0.00 & 0.06 & 0.38 & 0.44 & 0.17 & 0.42 & 0.43 & 0.34 & 0.48 & 0.19 & 0.44 & 0.37 & 0.08 & 0.01 & 0.20 & 0.22 \\
\hline $\begin{array}{c}\mathrm{Al}_{2} \mathrm{O}_{3} / \\
\mathrm{TiO}_{2}\end{array}$ & 4.75 & 4.88 & 13.78 & 55.17 & 18.85 & 25.74 & 17.29 & 19.86 & 16.03 & 24.35 & 91.67 & 33.58 & 61.57 & 7.50 & 12.20 & 18.98 & 30.40 \\
\hline $\begin{array}{c}\mathrm{Fe}_{2} \mathrm{O} / \\
\mathrm{K}_{2} \mathrm{O}\end{array}$ & 282.50 & 664.00 & 3.88 & 0.39 & 0.76 & 1.07 & 1.07 & 0.55 & 0.37 & 0.24 & 0.08 & 0.44 & 0.22 & 9.87 & 27.23 & 1.75 & 1.30 \\
\hline CIA & 98.92 & 99.50 & 93.47 & 67.91 & 68.41 & 85.66 & 68.88 & 64.31 & 74.19 & 66.67 & 83.52 & 63.01 & 72.00 & 91.98 & 98.56 & 75.30 & 56.93 \\
\hline PIA & 99.22 & 99.73 & 99.04 & 87.47 & 95.83 & 99.90 & 95.14 & 82.28 & 98.84 & 95.65 & 99.33 & 79.63 & 97.86 & 99.06 & 99.54 & 85.88 & 59.30 \\
\hline ICV & 1.12 & 1.73 & 0.40 & 0.65 & 0.93 & 0.43 & 1.06 & 0.97 & 0.59 & 0.71 & 0.23 & 0.91 & 0.50 & 1.00 & 0.37 & 0.83 & 1.20 \\
\hline CIW & 99.22 & 99.73 & 99.09 & 91.89 & 97.61 & 99.92 & 97.13 & 89.11 & 99.23 & 97.67 & 99.46 & 87.57 & 98.65 & 99.14 & 99.54 & 88.32 & 65.24 \\
\hline
\end{tabular}

LOI: Loss of ignition. CIA $(\%)=\left[\mathrm{Al}_{2} \mathrm{O}_{3} /\left(\mathrm{Al}_{2} \mathrm{O}_{3}+\mathrm{CaO}^{*}+\mathrm{Na}_{2} \mathrm{O}+\mathrm{K}_{2} \mathrm{O}\right)\right] \times 100$ from [21]. PIA (\%) $=\left[\mathrm{Al}_{2} \mathrm{O}_{3}-\mathrm{K}_{2} \mathrm{O} /\left(\mathrm{Al}_{2} \mathrm{O}_{3}+\mathrm{CaO}^{*}+\mathrm{Na}_{2} \mathrm{O}-\mathrm{K}_{2} \mathrm{O}\right)\right] \times 100$ from [21] and [45]. ICV $=\left(\mathrm{FeO}_{3}+\mathrm{K}_{2} \mathrm{O}+\mathrm{Na}_{2} \mathrm{O}+\mathrm{CaO}+\mathrm{MgO}+\mathrm{TiO}_{2}\right) / \mathrm{Al}_{2} \mathrm{O}_{3}$ from [46]. $\mathrm{CIW}=\left(\mathrm{Al}_{2} \mathrm{O}_{3} /\left(\mathrm{Al}_{2} \mathrm{O}_{3}+\mathrm{CaO}^{*}+\mathrm{Na}_{2} \mathrm{O}\right)\right] \times 100$ from [47]. The PAAS and UCC values are cited from [9] and [48]. 
Both arkose and $\mathrm{Fe}$-sandstone show similar composition compared to the rest. $\mathrm{SiO}_{2}$ and $\mathrm{Al}_{2} \mathrm{O}_{3}$ are the two abundant elements and are negatively correlated $(\mathrm{r}=$ -0.85). Arkose (67.3\% - 84.1\%) and Fe-sandstone (76.8\% - 79.8\%), are enriched in $\mathrm{SiO}_{2}$ compared to $\mathrm{Fe}$-shale $(24.6 \%-59.4 \%)$ and shale $(55 \%-65.1 \%)$, suggesting their higher quartz content. Fe-shale $(18.6 \%-25.6 \%)$ and shale $(14.9 \%$ 24.2\%) have higher contents of $\mathrm{Al}_{2} \mathrm{O}_{3}$, indicating their higher content in clay phases and other clay-sized phases. Both $\mathrm{K}_{2} \mathrm{O}(4.03 \%$ - 7.5\%) and $\mathrm{MgO}(0.22 \%$ $1.87 \%)$ are higher in shale. The concentrations of $\mathrm{CaO}, \mathrm{Na}_{2} \mathrm{O}$ and $\mathrm{P}_{2} \mathrm{O}_{5}$ are low and vary from $0 \%$ to $1.72 \%, 0.01 \%$ to $1.35 \%$ and $0.02 \%$ to $0.66 \%$ respectively. $\mathrm{Fe}_{2} \mathrm{O}_{3}$ is more concentrated in the Fe-shales $(22.6 \%-133.2 \%)$ than the other samples $(0.17 \%-7.52 \%)$.

Shale has $\mathrm{SiO}_{2}$ concentration similar to the average Post Archean Australian Shale (PAAS) (Figure 5(a)). Ignoring shale and arkose, $\mathrm{TiO}_{2}(1.07 \%-5.39 \%)$ has higher concentration compared to the average PAAS and Upper Continental Crust (UCC) (Figure 5(a) \& Figure 5(b)). Fe-shale and shale samples have higher concentrations of $\mathrm{Al}_{2} \mathrm{O}_{3}$ compared to the UCC and PAAS [9] [48]. All the samples have lower concentration of $\mathrm{MgO}, \mathrm{CaO}$ and $\mathrm{Na}_{2} \mathrm{O}$ than the UCC. Most of the arkose samples and all the $\mathrm{Fe}$-sandstone samples are highly depleted in $\mathrm{CaO}$. Most shale and arkose samples are enriched in $\mathrm{K}_{2} \mathrm{O}$. $\mathrm{MnO}$ shows a relative dispersion in abundance $(0.01 \%-0.11 \%)$. Except sample BB2, the content of $\mathrm{MnO}$ is, in all samples, well below both the PAAS and UCC values $(0.1 \%)$.
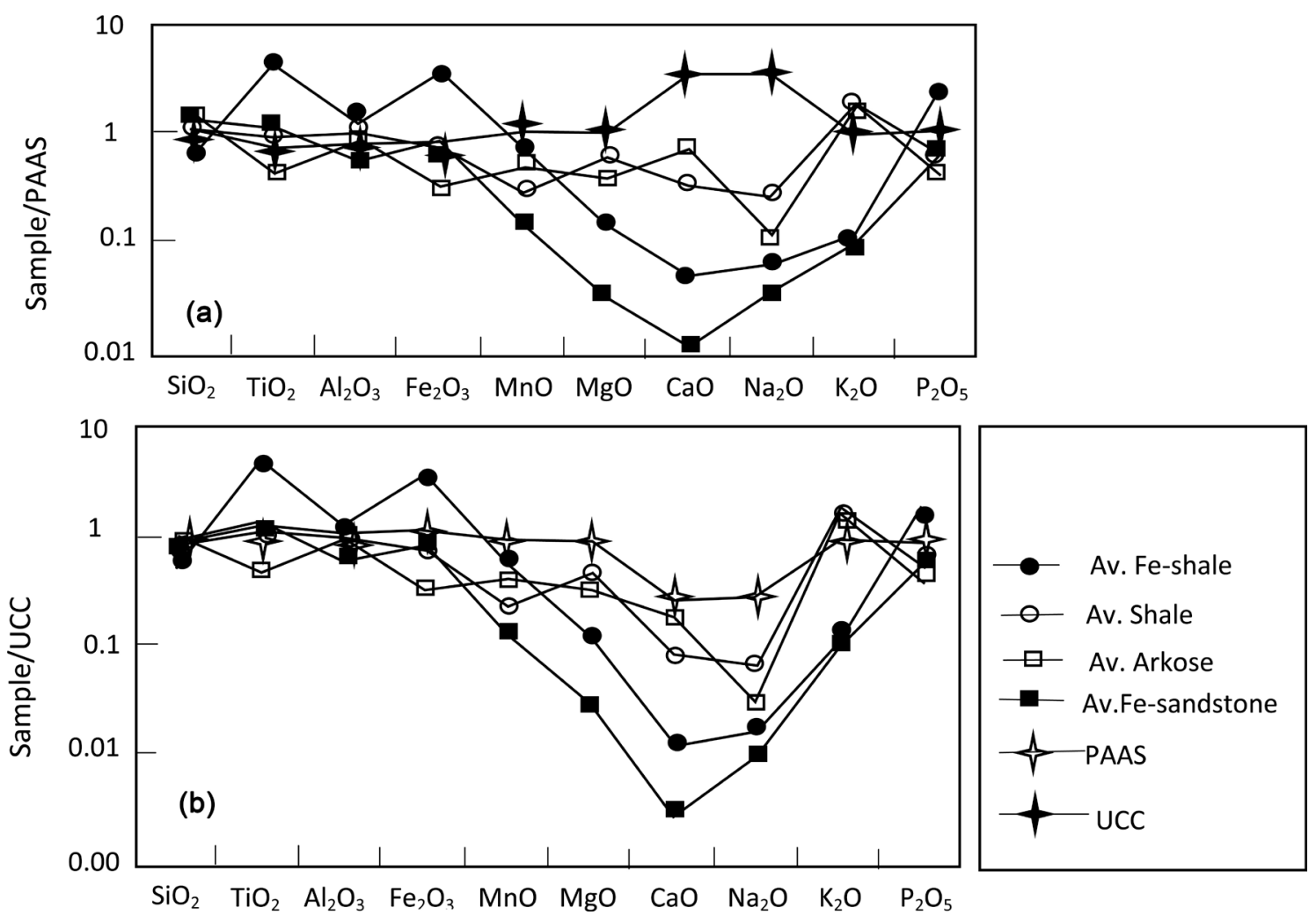

Figure 5. Distribution of major element oxides for average of various sediment samples: (a) PAAS-normalized, (b) UCC-normalized. The average composition of UCC and PAAS are considered from [9] and [48]. The plot of UCC and PAAS, on diagram (a) and (b) respectively, is shown for comparison. Av. = average. 


\subsection{Trace Elements}

The trace element concentrations and elemental ratios are presented in Table 2. The contents of $\mathrm{Ba}, \mathrm{Cr}, \mathrm{V}, \mathrm{Nb}, \mathrm{Sr}, \mathrm{Rb}$ and $\mathrm{Ni}$ show a wide range from 104 to $1870 \mathrm{ppm},<10$ to $600 \mathrm{ppm}, 11$ to $581 \mathrm{ppm}, 3.8$ to $86.2 \mathrm{ppm}, 43.5$ to $341 \mathrm{ppm}, 3.2$ to $256 \mathrm{ppm}, 6$ to $311 \mathrm{ppm}$ respectively. Shales show higher concentrations of Rb (134.5 - $256 \mathrm{ppm}), \mathrm{Sr}$ (170 - $341 \mathrm{ppm}), \mathrm{Ba}(138.5$ - $1870 \mathrm{ppm})$ and $\mathrm{Pb}(16-51$ ppm). Fe-shales have higher concentration of Cr (120 - $600 \mathrm{ppm}), \mathrm{V}$ (115 - 581 ppm), Ni (53 - 311 ppm), Y (28.1 - 86.3 ppm), Ga (27.5 - 39.5 ppm), Sc (16 - 37 ppm), Li (10 - $30 \mathrm{ppm}), \mathrm{Co}(23-27 \mathrm{ppm})$ and Ta (3.1 - 5.6 ppm). Shale sample TJC4 has the highest Zr content (1680) well above the PAAS and UCC contents (210 and 190 ppm respectively). Figure 6(a) \& Figure 6(b) presents the PAAS and UCC normalized trace element distributions in mudrocks and sandstones. Compare with PAAS, $\mathrm{Ta}, \mathrm{Nb}$ and $\mathrm{Zr}$ are enriched whereas $\mathrm{Cs}$ is distinctly depleted.

\subsection{Rare Earth Elements}

The rare earth element concentrations and elemental ratios are presented in Table 3. Total REE content varies between 81.17 and $738.75 \mathrm{ppm}$ with higher concentrations (289.67 - $738.75 \mathrm{ppm}$ ) in the Fe-shale and shale samples. La, Ce and Nd have higher contents than other REE, particularly in Fe-shale and shale. The total content of these three lanthanides vary between 69.20 and $543 \mathrm{ppm}$ with more than $233.10 \mathrm{ppm}$ in Fe-shale samples (Table 3). Amongst HREE, only Gd and Dy have relatively high concentrations (>7 ppm), particularly in the Feshale.

REE concentrations normalized to PAAS [9] [48] indicate: (i) REE-enrichment except in some sandstone samples (MTR1, TJC3 and BB5); (ii) LREEenrichment in Fe-shale, shale and some sandstone samples; (iii) HREE depletion in sandstone and one shale samples; (iv) positive $\mathrm{Eu}$ anomalies $\left(\mathrm{Eu} / \mathrm{Eu}^{*} \sim 1.04\right.$ to 1.56), particularly for the Fe-shale and shale; (v) slight negative Eu anomalies $\left(\mathrm{Eu} / \mathrm{Eu}^{*} \sim 0.87-0.98\right)$ for two sandstone samples; (vi) REE slight fractionation for both mudrocks and sandstones $\left((\mathrm{La} / \mathrm{Yb})_{\mathrm{N}}\right.$ varying between 0.60 and 3.99); and (vii) low $(\mathrm{La} / \mathrm{Sm})_{\mathrm{N}}$, varying between 0.56 and 1.72 , and variable $(\mathrm{Gd} / \mathrm{Yb})_{\mathrm{N}}$ ratios, ranging from 0.78 to 3.55 ; (viii) $\mathrm{Ce} / \mathrm{Ce}^{\star}$ ranges from 0.89 to 1.03 with lesser values in the Fe-shale (Table 3; Figure 7(a)). For comparison, REE compositions of the studied samples and the PAAS were normalized to the chondrite [49] (Figure 7(b)). The chondrite-normalised REE pattern confirms broadly the similar LREE-enrichment for the studied samples and the slight negative Eu anomalies for average sandstone samples (Figures 8(a)-(d)). Figure 9 presents the chondrite normalized REE pattern [49] of the probable source rocks.

\section{Discussion}

\subsection{Paleoweathering and Diagenesis}

Weathering of parent-rocks consist mainly of the removal of alkaline and alka- 
Table 2. Trace element composition (ppm) and element ratios of Kumba Cretaceous sediments and average compositions of PASS and UCC.

\begin{tabular}{|c|c|c|c|c|c|c|c|c|c|c|c|c|c|c|c|c|c|}
\hline \multirow{2}{*}{$\begin{array}{c}\text { Sample } \\
\text { Ref. code }\end{array}$} & \multicolumn{3}{|c|}{$\underline{\text { Fe-shale }}$} & \multicolumn{6}{|c|}{ Shale } & \multicolumn{4}{|c|}{ Arkose } & \multicolumn{2}{|c|}{$\underline{\text { Fe-sandstone }}$} & \multirow[b]{2}{*}{ PAAS } & \multirow[b]{2}{*}{ UCC } \\
\hline & BB2 & BB3 & BB4 & EDDd & MTR5 & MTR4 & TJC1 & TJC2 & TJC4 & $\mathrm{MTFb}$ & MTR1 & TJC3 & TJC5 & BB5 & BB1 & & \\
\hline $\mathrm{Ba}$ & 194.00 & 146.50 & 387.00 & 1505.00 & 1220.00 & 138.50 & 1020.00 & 1090.00 & 1870.00 & 1345.00 & 534.00 & 1265.00 & 1110.00 & 193.50 & 104.00 & 650.00 & 550.00 \\
\hline $\mathrm{Cr}$ & 390.00 & 600.00 & 120.00 & 20.00 & 70.00 & 110.00 & 90.00 & 60.00 & 80.00 & 40.00 & $<10$ & 40.00 & 20.00 & 160.00 & 60.00 & 110.00 & 83.00 \\
\hline $\mathrm{Zr}$ & 371.00 & 362.00 & 348.00 & 110.00 & 567.00 & 242.00 & 442.00 & 336.00 & 1680.00 & 721.00 & 110.00 & 196.00 & 171.00 & 371.00 & 531.00 & 210.00 & 190.00 \\
\hline $\mathrm{Th}$ & 7.71 & 8.74 & 16.55 & 10.60 & 30.20 & 26.30 & 25.10 & 1.15 & 50.50 & 32.30 & 6.04 & 9.91 & 8.83 & 9.15 & 15.10 & 14.60 & 10.70 \\
\hline $\mathrm{U}$ & 2.06 & 3.51 & 7.22 & 2.89 & 3.24 & 4.00 & 3.09 & 3.69 & 5.96 & 3.75 & 1.02 & 2.14 & 1.9 .00 & 2.74 & 2.01 & 3.10 & 2.80 \\
\hline $\mathrm{V}$ & 460.00 & 581.00 & 115.00 & 39.00 & 68.00 & 75.00 & 82.00 & 71.00 & 81.00 & 41.00 & 11.00 & 47.00 & 19.00 & 108.00 & 91.00 & 150.00 & 107.00 \\
\hline $\mathrm{Hf}$ & 8.80 & 8.80 & 8.40 & 3.30 & 16.60 & 7.00 & 11.50 & 9.30 & 44.50 & 20.00 & 3.40 & 4.80 & 4.70 & 8.60 & 13.40 & 5.00 & 5.80 \\
\hline $\mathrm{Nb}$ & 86.20 & 80.40 & 45.50 & 11.10 & 20.90 & 21.50 & 22.70 & 16.60 & 26.20 & 16.10 & 3.80 & 8.50 & 5.20 & 29.50 & 35.60 & 19.00 & 12.00 \\
\hline $\mathrm{Y}$ & 28.10 & 33.50 & 86.30 & 8.80 & 33.10 & 116.00 & 35.40 & 22.50 & 67.70 & 26.40 & 12.70 & 21.00 & 7.30 & 10.90 & 19.60 & 27.00 & 22.00 \\
\hline $\mathrm{Ta}$ & 5.60 & 5.00 & 3.10 & 0.80 & 1.50 & 1.60 & 1.60 & 1.30 & 2.20 & 1.30 & 0.40 & 0.60 & 0.40 & 1.90 & 2.40 & 1.28 & 1.00 \\
\hline $\mathrm{Ga}$ & 39.50 & 36.40 & 27.50 & 17.00 & 25.30 & 35.40 & 24.40 & 19.40 & 25.40 & 19.30 & 11.20 & 14.90 & 9.30 & 13.10 & 19.00 & 20.00 & 17.00 \\
\hline $\mathrm{Rb}$ & 3.50 & 3.20 & 57.00 & 158.50 & 256.00 & 134.50 & 233.00 & 191.50 & 190.50 & 213.00 & 66.10 & 152.00 & 114.50 & 24.10 & 12.10 & 160.00 & 112.00 \\
\hline $\mathrm{Ni}$ & 311.00 & 179.00 & 53.00 & 33.00 & 29.00 & 39.00 & 33.00 & 31.00 & 22.00 & 16.00 & 14.00 & 12.00 & 6.00 & 21.00 & 25.00 & 55.00 & 44.00 \\
\hline Co & 26.00 & 23.00 & 27.00 & 21.00 & 9.00 & 10.00 & 8.00 & 10.00 & 5.00 & 5.00 & 32.00 & 7.00 & 1.00 & 4.00 & 2.00 & 23.00 & 17.00 \\
\hline $\mathrm{Cu}$ & 78.00 & 89.00 & 34.00 & 18.00 & 3.00 & 13.00 & $<1$ & 25.00 & 5.00 & 2.00 & 6.00 & 7.00 & 1.00 & 14.00 & 9.00 & 50.00 & 25.00 \\
\hline $\mathrm{Sc}$ & 37.00 & 37.00 & 16.00 & 3.00 & 9.00 & 12.00 & 11.00 & 9.00 & 10.00 & 6.00 & 1.00 & 6.00 & 2.00 & 9.00 & 9.00 & 16.00 & 13.60 \\
\hline $\mathrm{Pb}$ & $<2$ & $<2$ & 22.00 & 51.00 & 26.00 & 16.00 & 25.00 & 25.00 & 48.00 & 22.00 & 31.00 & 25.00 & 20.00 & 8.00 & 13.00 & 20.00 & 17.00 \\
\hline $\mathrm{Zn}$ & 187.00 & 120.00 & 57.00 & 8.00 & 54.00 & 67.00 & 63.00 & 40.00 & 37.00 & 29.00 & 5.00 & 28.00 & $<2$ & 23.00 & 22.00 & 85.00 & 71.00 \\
\hline $\mathrm{Li}$ & 10.00 & 10.00 & 30.00 & $<10$ & - & 10.00 & $<10$ & $<10$ & 10.00 & $<10$ & $<10$ & 10.00 & 10.00 & 10.00 & 10.00 & - & - \\
\hline $\mathrm{Ti}$ & $<10$ & $<10$ & $<10$ & $<10$ & 10.00 & $<10$ & $<10$ & $<10$ & $<10$ & $<10$ & $<10$ & $<10$ & $<10$ & $<10$ & $<10$ & - & - \\
\hline $\mathrm{Zr} / \mathrm{Hf}$ & 42.16 & 41.14 & 41.43 & 33.33 & 34.16 & 34.57 & 38.43 & 36.13 & 37.75 & 36.05 & 32.35 & 40.83 & 36.38 & 43.14 & 39.63 & 42.00 & 32.80 \\
\hline $\mathrm{Cr} / \mathrm{Th}$ & 50.58 & 68.65 & 7.25 & 1.89 & 2.32 & 4.18 & 3.59 & 52.17 & 1.58 & 1.24 & - & 4.04 & 2.27 & 17.49 & 3.97 & 7.53 & 7.76 \\
\hline $\mathrm{Cr} / \mathrm{Ni}$ & 1.25 & 3.35 & 2.26 & 0.61 & 2.41 & 2.82 & 2.73 & 1.94 & 3.64 & 2.50 & - & 3.33 & 3.33 & 7.62 & 2.40 & 2.00 & 1.89 \\
\hline $\mathrm{V} / \mathrm{Cr}$ & 1.18 & 0.97 & 0.96 & 1.95 & 0.97 & 0.68 & 0.91 & 1.18 & 1.01 & 1.03 & - & 1.18 & 0.95 & 0.68 & 1.52 & 1.36 & 1.29 \\
\hline $\mathrm{Th} / \mathrm{Cr}$ & 0.02 & 0.01 & 0.14 & 0.53 & 0.43 & 0.24 & 0.28 & 0.02 & 0.63 & 0.81 & - & 0.25 & 0.44 & 0.06 & 0.25 & 0.13 & 0.13 \\
\hline $\mathrm{Th} / \mathrm{U}$ & 3.74 & 2.49 & 2.29 & 3.67 & 9.32 & 6.58 & 8.12 & 0.31 & 8.47 & 8.61 & 5.92 & 4.63 & 8.10 & 3.34 & 7.51 & 4.71 & 3.82 \\
\hline Th/Co & 0.30 & 0.38 & 0.61 & 0.50 & 3.36 & 2.63 & 3.14 & 0.12 & 10.10 & 6.46 & 0.19 & 1.42 & 8.83 & 2.29 & 7.55 & 0.63 & 0.63 \\
\hline $\mathrm{Th} / \mathrm{Sc}$ & 0.21 & 0.24 & 1.03 & 3.53 & 3.36 & 2.19 & 2.28 & 0.13 & 5.05 & 5.38 & 6.04 & 1.65 & 4.42 & 1.02 & 1.68 & 0.91 & 0.79 \\
\hline $\mathrm{U} / \mathrm{Th}$ & 0.27 & 0.40 & 0.44 & 0.27 & 0.11 & 0.15 & 0.12 & 3.21 & 0.12 & 0.12 & 0.17 & 0.22 & 0.12 & 0.30 & 0.13 & 0.21 & 0.26 \\
\hline $\mathrm{Ni} / \mathrm{Co}$ & 11.96 & 7.78 & 1.96 & 1.57 & 3.22 & 3.90 & 4.13 & 3.10 & 4.40 & 3.20 & 0.44 & 1.71 & 6.00 & 5.25 & 12.50 & 1.10 & 2.59 \\
\hline $\mathrm{Cu} / \mathrm{Zn}$ & 0.42 & 0.74 & 0.60 & 2.25 & 0.06 & 0.19 & 0.01 & 0.63 & 0.13 & 0.07 & 1.20 & 0.25 & 0.50 & 0.61 & 0.41 & 0.58 & 0.35 \\
\hline $\mathrm{Y} / \mathrm{Ni}$ & 0.09 & 0.19 & 1.63 & 0.27 & 1.14 & 2.97 & 1.07 & 0.73 & 3.08 & 1.65 & 0.91 & 1.75 & 1.22 & 0.52 & 0.78 & 0.49 & 0.50 \\
\hline $\mathrm{Co} / \mathrm{Th}$ & 3.37 & 2.63 & 1.63 & 1.98 & 0.30 & 0.38 & 0.32 & 8.70 & 0.10 & 0.15 & 5.30 & 0.71 & 0.11 & 0.44 & 0.13 & 1.58 & 1.59 \\
\hline $\mathrm{Zr} / \mathrm{Cr}$ & 0.95 & 0.60 & 2.90 & 5.50 & 8.10 & 2.20 & 4.91 & 5.60 & 21.00 & 1.03 & - & 4.90 & 8.55 & 2.32 & 8.85 & 1.91 & 2.29 \\
\hline $\mathrm{Zr} / \mathrm{Sc}$ & 10.03 & 9.78 & 21.75 & 36.67 & 63.00 & 20.17 & 40.18 & 37.33 & 168.00 & 120.17 & 110.00 & 32.67 & 85.50 & 41.22 & 59.00 & 13.13 & 13.97 \\
\hline $\mathrm{Cr} / \mathrm{V}$ & 0.85 & 1.03 & 1.04 & 0.51 & 1.03 & 1.47 & 1.10 & 0.85 & 0.99 & 0.98 & - & 0.85 & 1.05 & 1.48 & 0.66 & 0.73 & 0.78 \\
\hline $\mathrm{Cr} / \mathrm{Zr}$ & 1.05 & 1.65 & 0.34 & 0.18 & 0.12 & 0.45 & 0.20 & 0.18 & 0.05 & 0.06 & - & 0.20 & 0.12 & 0.43 & 0.11 & 0.52 & 0.43 \\
\hline
\end{tabular}

The PAAS and UCC values are cited from [9] and [48]. 
Table 3. Rare-earth element composition (ppm) and element ratios of Kumba Cretaceous sediments and average compositions of PASS and UCC.

\begin{tabular}{|c|c|c|c|c|c|c|c|c|c|c|c|c|c|c|c|c|c|}
\hline \multirow{2}{*}{$\begin{array}{c}\text { Sample } \\
\text { Ref. code }\end{array}$} & \multirow[b]{2}{*}{ BB2 } & \multicolumn{2}{|l|}{$\underline{\text { Fe-shale }}$} & \multicolumn{5}{|c|}{$\underline{\text { Shale }}$} & \multicolumn{5}{|c|}{$\underline{\text { Arkose }}$} & \multicolumn{2}{|c|}{$\underline{\text { Fe-sandstone }}$} & \multirow[b]{2}{*}{ PAAS } & \multirow[b]{2}{*}{ UCC } \\
\hline & & BB3 & BB4 & EDDd & MTR5 & MTR4 & TJC1 & TJC2 & TJC4 & $\mathrm{MTFb}$ & MTR1 & TJC3 & TJC5 & BB5 & $\mathrm{BB} 1$ & & \\
\hline $\mathrm{La}$ & 67.20 & 53.70 & 117.00 & 47.40 & 64.00 & 65.70 & 67.70 & 49.70 & 182.50 & 51.40 & 19.90 & 26.60 & 19.80 & 29.20 & 48.90 & 38.20 & 30.00 \\
\hline $\mathrm{Ce}$ & 139.00 & 112.50 & 287.00 & 93.10 & 126.00 & 126.50 & 127.50 & 92.20 & 316.00 & 101.50 & 36.50 & 48.70 & 37.30 & 52.50 & 106.00 & 79.60 & 64.00 \\
\hline $\operatorname{Pr}$ & 19.50 & 15.95 & 35.20 & 10.20 & 14.05 & 12.45 & 14.55 & 10.65 & 37.20 & 11.10 & 3.83 & 5.66 & 3.98 & 6.08 & 11.70 & 8.83 & 7.10 \\
\hline $\mathrm{Nd}$ & 78.00 & 66.90 & 139.00 & 38.00 & 52.90 & 42.40 & 52.20 & 40.50 & 130.00 & 40.80 & 12.80 & 21.80 & 15.70 & 21.70 & 45.20 & 33.90 & 26.00 \\
\hline $\mathrm{Sm}$ & 16.35 & 13.90 & 25.00 & 5.74 & 8.18 & 6.26 & 9.25 & 7.19 & 22.30 & 5.70 & 1.68 & 4.14 & 2.86 & 4.29 & 8.80 & 5.55 & 4.50 \\
\hline $\mathrm{Eu}$ & 4.68 & 4.13 & 5.74 & 1.74 & 1.77 & 1.71 & 1.96 & 1.52 & 4.82 & 1.18 & 0.42 & 1.03 & 0.78 & 0.67 & 1.75 & 1.08 & 0.88 \\
\hline $\mathrm{Gd}$ & 12.20 & 12.00 & 20.20 & 4.48 & 7.85 & 9.02 & 6.63 & 5.37 & 14.65 & 5.65 & 1.51 & 3.11 & 2.03 & 3.06 & 6.90 & 4.66 & 3.80 \\
\hline $\mathrm{Tb}$ & 1.68 & 1.54 & 2.75 & 0.45 & 0.93 & 1.41 & 1.02 & 0.73 & 2.27 & 0.68 & 0.24 & 0.45 & 0.32 & 0.45 & 0.98 & 0.77 & 0.64 \\
\hline Dy & 8.05 & 8.01 & 16.15 & 2.13 & 5.86 & 12.20 & 5.46 & 4.23 & 12.75 & 4.48 & 1.85 & 2.67 & 1.48 & 2.25 & 4.66 & 4.68 & 3.50 \\
\hline Ho & 1.20 & 1.24 & 2.98 & 0.37 & 1.09 & 2.83 & 1.15 & 0.76 & 2.24 & 0.89 & 0.34 & 0.58 & 0.26 & 0.42 & 0.76 & 0.99 & 0.80 \\
\hline $\mathrm{Er}$ & 2.70 & 3.29 & 8.09 & 0.83 & 3.26 & 8.96 & 3.37 & 2.04 & 6.39 & 2.50 & 1.02 & 1.85 & 0.74 & 1.13 & 2.04 & 2.85 & 2.30 \\
\hline $\mathrm{Tm}$ & 0.32 & 0.44 & 1.09 & 0.13 & 0.45 & 1.21 & 0.46 & 0.31 & 0.87 & 0.39 & 0.15 & 0.25 & 0.09 & 0.20 & 0.31 & 0.41 & 0.33 \\
\hline $\mathrm{Yb}$ & 2.08 & 2.30 & 6.26 & 0.88 & 2.89 & 6.98 & 3.39 & 1.89 & 5.86 & 2.45 & 0.81 & 1.54 & 0.66 & 1.10 & 2.02 & 2.82 & 2.20 \\
\hline $\begin{array}{c}\mathrm{La}+\mathrm{Ce}+ \\
\mathrm{Nd}\end{array}$ & 284.20 & 233.10 & 543.00 & 178.50 & 242.90 & 234.60 & 247.40 & 182.40 & 628.50 & 193.70 & 69.20 & 97.10 & 72.80 & 103.40 & 200.10 & 151.70 & 120.00 \\
\hline$\sum \mathrm{REE}$ & 353.21 & 296.22 & 667.38 & 205.56 & 289.67 & 298.71 & 295.12 & 217.39 & 738.75 & 229.12 & 81.17 & 118.59 & 86.10 & 123.22 & 240.28 & 184.77 & 146.37 \\
\hline$\sum$ LREE & 324.73 & 267.08 & 608.94 & 196.18 & 266.90 & 255.02 & 273.16 & 201.76 & 692.82 & 211.68 & 75.13 & 107.93 & 80.42 & 114.44 & 222.35 & 160.53 & 132.48 \\
\hline$\sum$ HREE & 28.48 & 29.14 & 58.44 & 9.38 & 22.77 & 43.69 & 21.96 & 15.63 & 45.93 & 17.44 & 6.04 & 10.66 & 5.68 & 8.78 & 17.93 & 12.18 & 13.89 \\
\hline $\begin{array}{c}\text { LREE/H } \\
\text { REE }\end{array}$ & 11.40 & 9.17 & 10.42 & 20.91 & 11.72 & 5.84 & 12.44 & 12.91 & 15.08 & 12.14 & 12.44 & 10.12 & 14.16 & 13.03 & 12.40 & 13.18 & 9.54 \\
\hline $\mathrm{Eu} / \mathrm{Eu}^{*}$ & 1.56 & 1.51 & 1.20 & 1.62 & 1.04 & 1.07 & 1.18 & 1.15 & 1.26 & 0.98 & 1.24 & 1.35 & 1.52 & 0.87 & 1.06 & 0.63 & 0.65 \\
\hline $\mathrm{Ce} / \mathrm{Ce}^{*}$ & 0.89 & 0.89 & 1.03 & 0.98 & 0.97 & 1.02 & 0.94 & 0.92 & 0.88 & 0.98 & 0.96 & 0.92 & 0.97 & 0.91 & 1.02 & 1.04 & 1.08 \\
\hline $\mathrm{La} / \mathrm{Th}$ & 8.72 & 6.14 & 7.07 & 4.47 & 2.12 & 2.50 & 8.78 & 5.69 & 11.03 & 1.59 & 3.29 & 2.51 & 0.66 & 3.19 & 3.24 & 2.31 & 2.80 \\
\hline $\mathrm{La} / \mathrm{Sc}$ & 1.82 & 1.45 & 7.31 & 15.80 & 7.11 & 5.48 & 1.83 & 1.34 & 11.41 & 8.57 & 19.90 & 8.87 & 2.20 & 3.24 & 5.43 & 2.39 & 2.20 \\
\hline$(\mathrm{La} / \mathrm{Yb}) \mathrm{n}$ & 2.39 & 1.72 & 1.38 & 3.98 & 1.93 & 0.69 & 1.47 & 1.94 & 2.30 & 1.55 & 1.81 & 1.28 & 0.60 & 1.96 & 1.79 & 9.15 & 9.21 \\
\hline$(\mathrm{La} / \mathrm{Sm}) \mathrm{n}$ & 0.60 & 0.56 & 0.68 & 1.20 & 1.14 & 1.17 & 1.06 & 1.00 & 1.19 & 1.31 & 1.72 & 0.93 & 1.01 & 0.99 & 0.81 & 4.33 & 4.20 \\
\hline$(\mathrm{Gd} / \mathrm{Yb}) \mathrm{n}$ & 3.55 & 3.16 & 1.95 & 3.08 & 1.64 & 0.78 & 1.18 & 1.72 & 1.51 & 1.40 & 1.13 & 1.22 & 1.86 & 1.68 & 2.07 & 1.34 & 1.40 \\
\hline
\end{tabular}

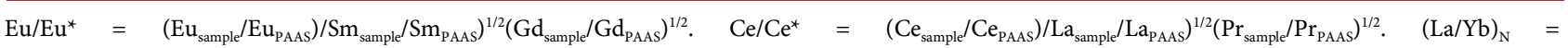
$\left(\mathrm{La}_{\text {sample }} / \mathrm{La}_{\mathrm{PAAS}_{\mathrm{S}}}\right) /\left(\mathrm{Yb}_{\text {sample }} / \mathrm{Yb}_{\mathrm{PAAS}}\right) \cdot(\mathrm{La} / \mathrm{Sm})_{\mathrm{N}}=\left(\mathrm{La}_{\text {sample }} / \mathrm{La}_{\mathrm{PAAS}}\right) /\left(\mathrm{Sm}_{\text {sample }} / \mathrm{Sm}_{\mathrm{PAAS}}\right) \cdot(\mathrm{Gd} / \mathrm{Yb})_{\mathrm{N}}=\left(\mathrm{Gd}_{\text {sample }} / \mathrm{Gd}_{\text {PAAS }}\right) /\left(\mathrm{Yb}_{\text {sample }} / \mathrm{Yb}_{\text {PAAS }}\right)$. The PAAS and UCC values are cited from [9] and [48]. 


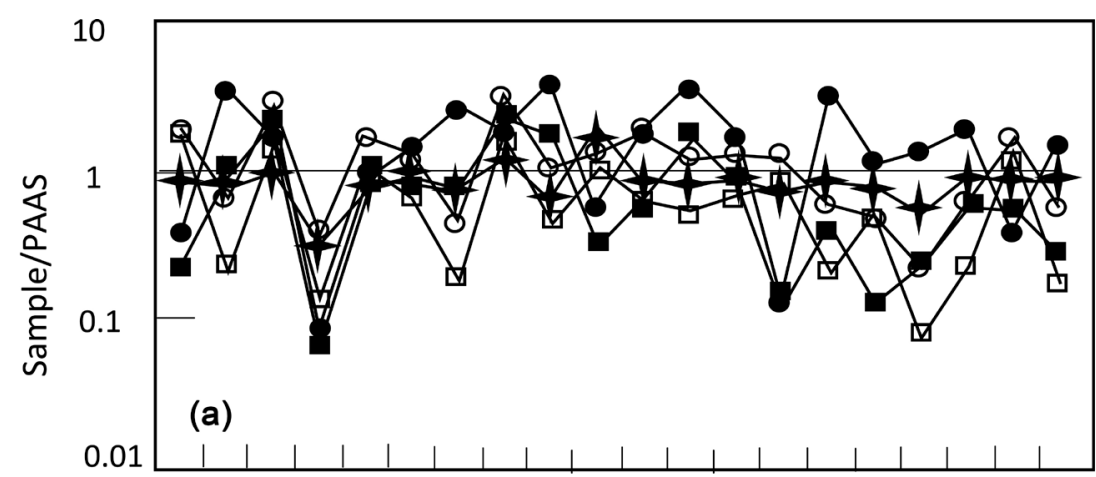

Ba Cr Zr Cs Th U V Hf Nb Sr Y Ta Ga Rb Ni Co Cu Sc Pb Zn
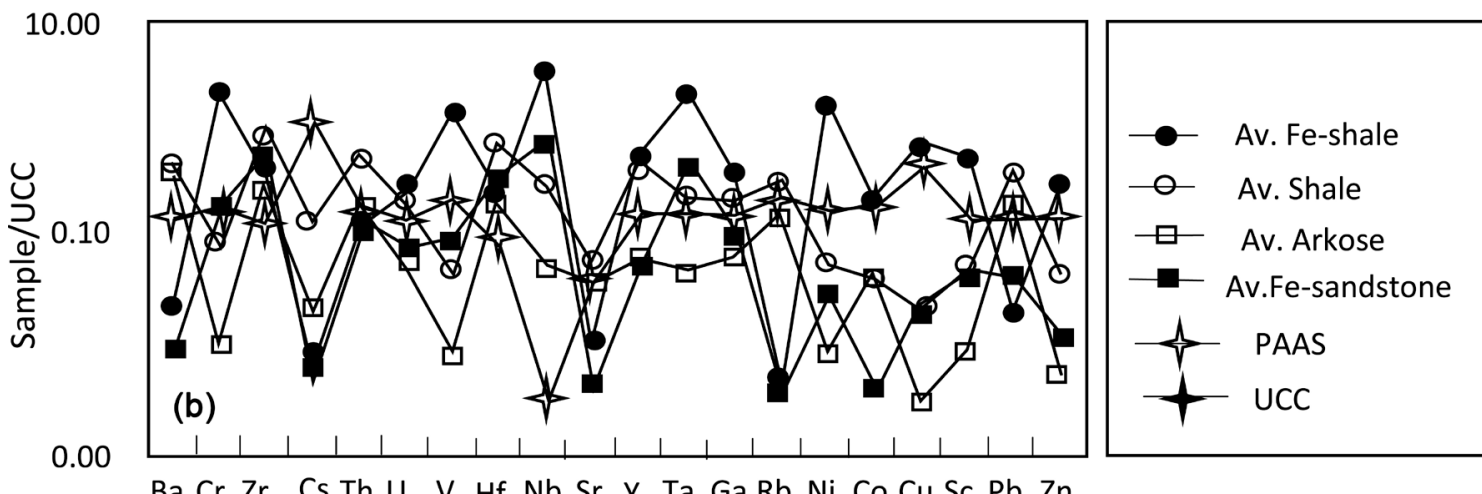

$\mathrm{Ba} \mathrm{Cr} \mathrm{Zr}$ Cs Th U V Hf Nb Sr Y Ta Ga Rb Ni Co Cu Sc Pb Zn

Figure 6. Distribution of trace elements for average of various sediments samples: (a) PAAS-normalized, (b) UCC-normalized. The average composition of UCC and PAAS are considered from [9] and [48]. The plot of UCC and PAAS, on diagram (a) and (b) respectively, is shown for comparison.
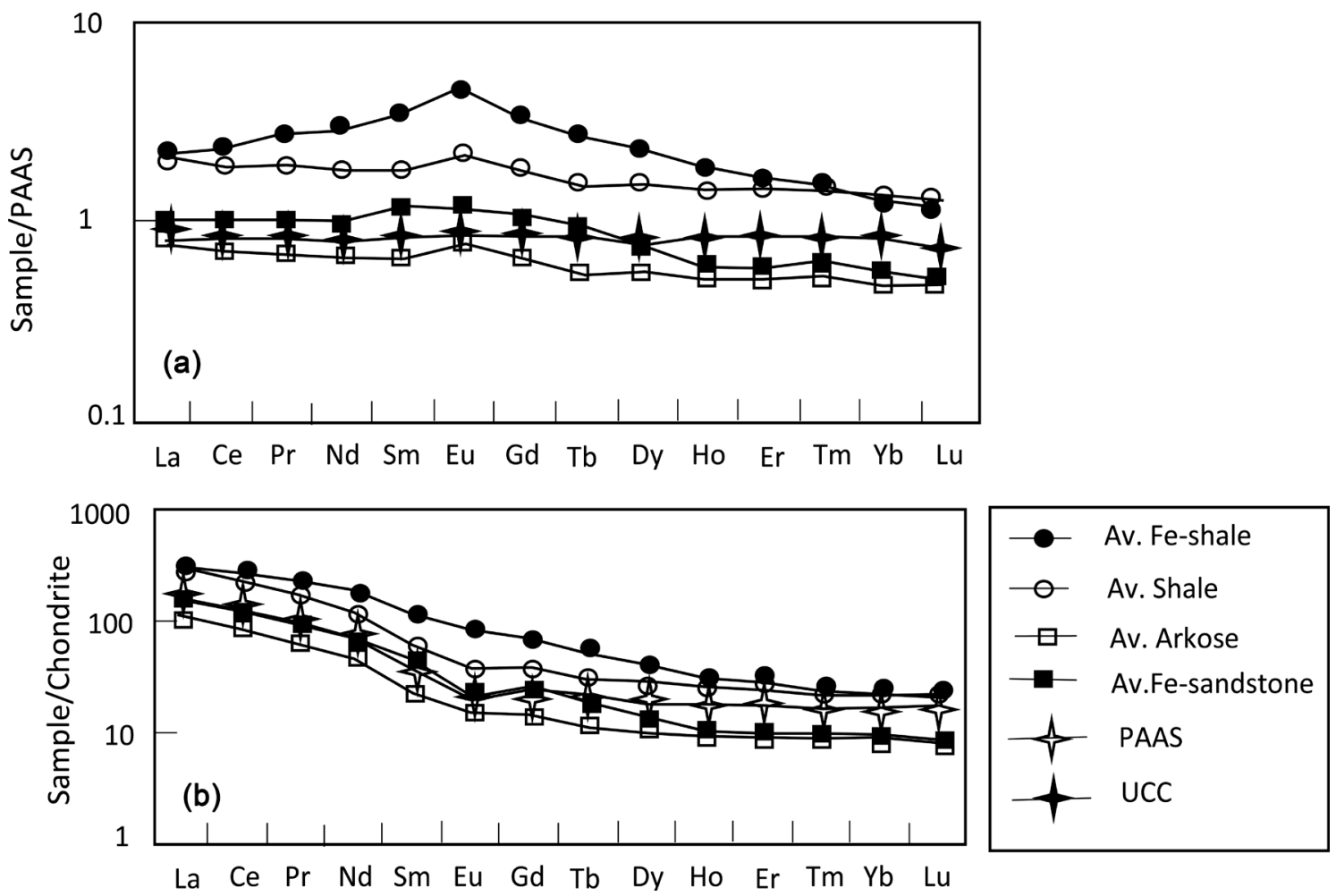

Figure 7. REE pattern for averages of different sediment samples: (a) PAAS-normalized [9] and [48], (b) chondrite-normalized [49]. The plot of UCC and PAAS on diagram (a) and (b) respectively is shown for comparison. 

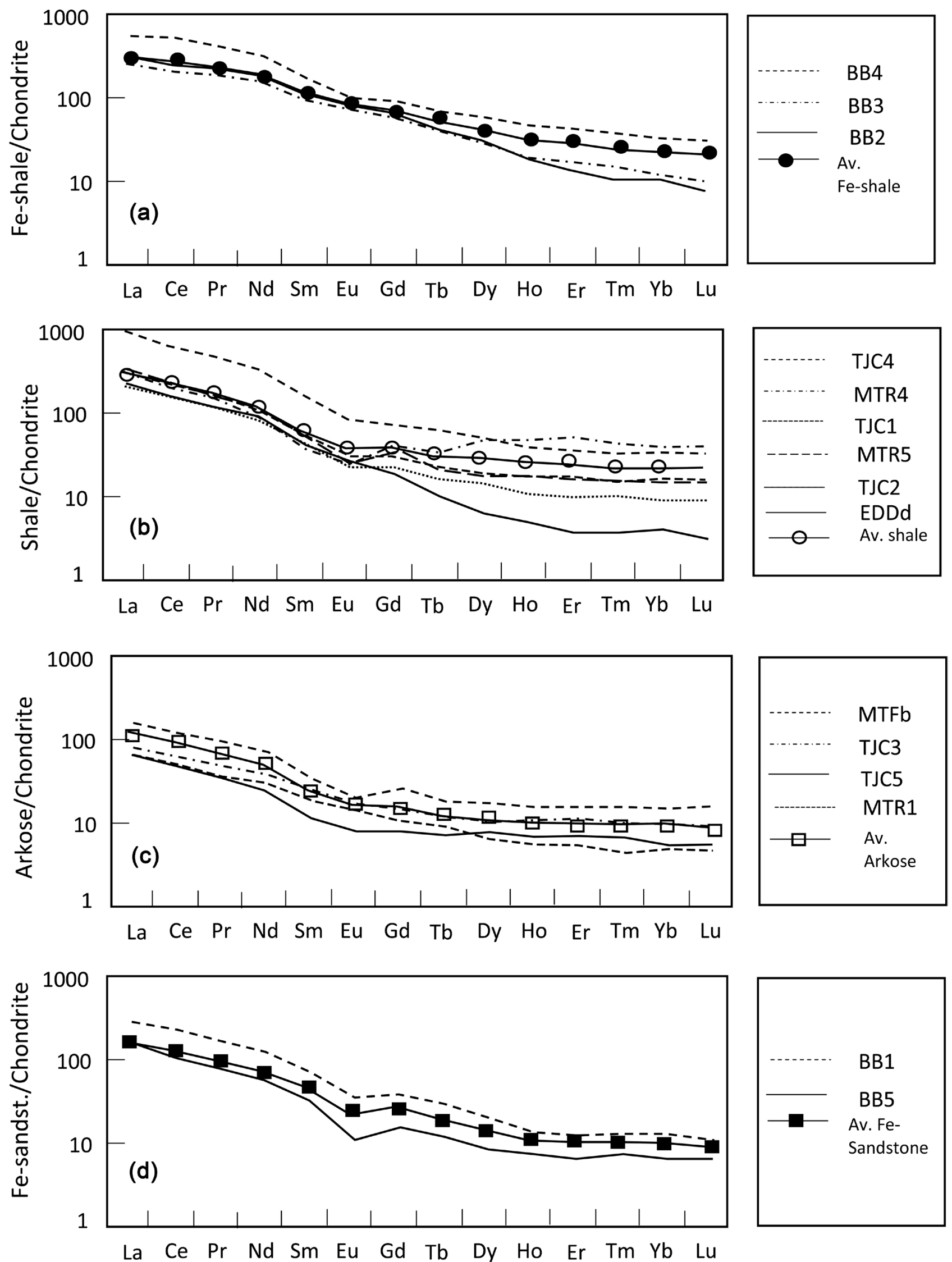

Figure 8. Chondrite-normalized REE plot for: (a) Fe-shale, (b) shale, (c) arkose, and (d) Fe-sandstone samples. 


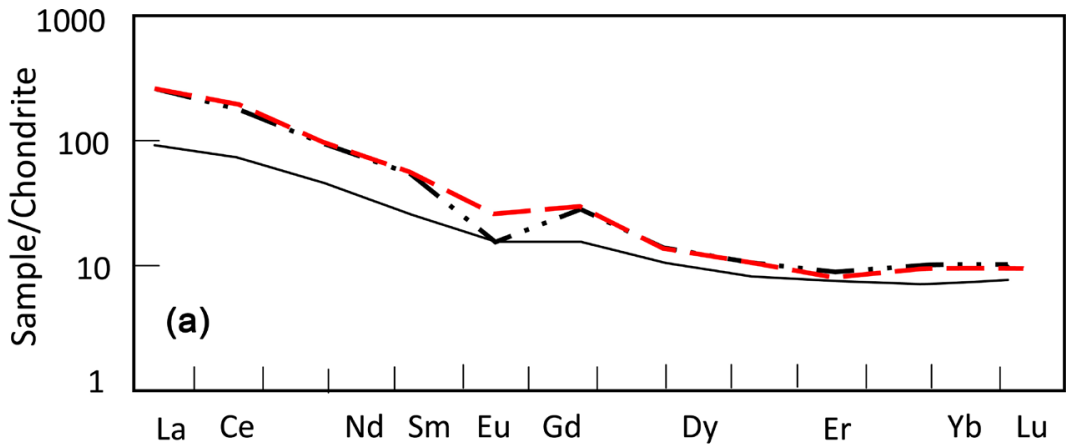

- - Av.Coarse-grained granite (CGG): $n=8$

_. - Av. Fine-grained granite (FGG): $n=8$ Av. Mafic to felsic $\operatorname{rock}(M F R): n=6$
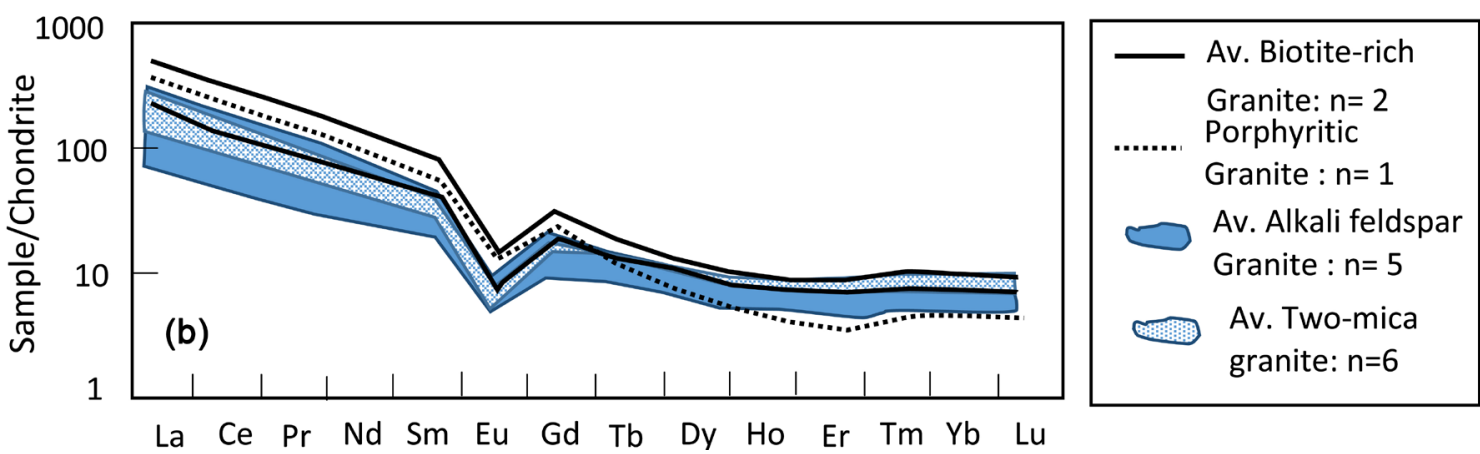

Figure 9. Chondrite normalized REE pattern [49] of the probable source rocks. REE data are from (a) [34] and (b) [35]. $\mathrm{n}=$ number of samples.

line-earth elements in siliclastic sediments [50]. The concentration of $\mathrm{Al}_{2} \mathrm{O}_{3}(\mathrm{~A})$, $\mathrm{CaO}$ and $\mathrm{Na}_{2} \mathrm{O}(\mathrm{CN})$ and $\mathrm{K}_{2} \mathrm{O}(\mathrm{K})$ is thus important for evaluating the geochemical alteration of the sediments. To this end, several chemical indices, using those concentrations, have been proposed. Amongst these indices, the Chemical Index of Alteration (CIA) is the most commonly used. CIA is defined as $\left[\mathrm{Al}_{2} \mathrm{O}_{3} /\left(\mathrm{Al}_{2} \mathrm{O}_{3}\right.\right.$ $\left.\left.+\mathrm{CaO}^{*}+\mathrm{Na}_{2} \mathrm{O}+\mathrm{K}_{2} \mathrm{O}\right)\right] \times 100$ where $\mathrm{CaO}^{*}$ is $\mathrm{Ca}$ exclusive of carbonates and the values are in molar proportions to emphasise mineralogical relationships [21] [47] [51] [52]. $\mathrm{CaO}$ was low in all considered samples. Therefore, $\mathrm{CaO}^{*}$ was regarded equal to $\mathrm{CaO}$. The Plagioclase Index of Alteration (PIA) $=\left[\mathrm{Al}_{2} \mathrm{O}_{3}-\right.$ $\left.\mathrm{K}_{2} \mathrm{O} /\left(\mathrm{Al}_{2} \mathrm{O}_{3}+\mathrm{CaO}^{*}+\mathrm{Na}_{2} \mathrm{O}-\mathrm{K}_{2} \mathrm{O}\right)\right] \times 100$ is also sensitive to chemical alteration of sediments [21] [45]. Both the CIA and the PIA provide the quantitative estimations of secondary aluminous clay mineral abundance with respect to the primary feldspar [21]. High values of CIA and PIA (i.e., 76 - 100) are indicative of intense chemical weathering in the source areas whereas low values (i.e., 50 or less) reflect unweathered source areas. For comparison and to facilitate interpretation, PAAS and UCC have PIA values of 85.88 and 59.30, respectively. CIA values of Fe-shale (93.47 - 99.50), shale (67.91 - 85.66), arkose (66.67 - 83.52) and Fe sandstone (91.98 - 98.56) suggest moderate to extreme chemical weathering (Table 1). The PIA values $(87.47$ - 99.90) are always high, indicating probably the degradation of almost all the plagioclase feldspar into clays minerals.

The weathering and association of detrital mineralogy can also be constrained by calculating the Index of Compositional Variability $\left[\mathrm{ICV}=\left(\mathrm{FeO}_{3}+\mathrm{K}_{2} \mathrm{O}+\right.\right.$ $\left.\mathrm{Na}_{2} \mathrm{O}+\mathrm{CaO}+\mathrm{MgO}+\mathrm{TiO}_{2}\right) / \mathrm{Al}_{2} \mathrm{O}_{3}$ ] (Table 1) [46]. Immature sediments, containing a high proportion of silicates other than clays, commonly show high val- 
ues of this index (ICV >1), whereas mature sediments, depleted in silicates other than clays, generally show low ICV values $(\mathrm{ICV}<1)$. The ICV values of Kumba mudrocks and sandstones vary between 0.23 and 1.73 (Table 1) with only a few exceptions $>1$. This suggests the presence of dominant clay mineral such as kaolinite and illite, indicating intense weathering of first-cycle sediments or recycled sediments [53]; but also, to a lesser extent, the rock forming mineral such as K-feldspar.

To quantify weathering effects, the analyzed samples are plotted in the A-CN-K ternary plot of Nesbitt and Young [54] (Figure 10). On this triangular diagram, most of the samples are aligned between the neighborhood of the muscovite point and the A apex which is the idealized fields of the clay minerals such as kaolinite and chlorite. This feature reflects intense weathering but also probably diagenetic processes such as $\mathrm{K}$-addition, since kaolinite as the main clay mineral may undergo K-metasomatism to produce illite. As a result, such samples will plot very close to the A-K boundary. Moreover, they can form a trend at right angles to the A-K joint [45] [55]. This is almost the case for some of the studied samples; few shale and arkose samples plotting near the muscovite point exhibit a clear cut trend back, at right angles to the A-K joint (heavy solid line; Figure 10) indicating probably $\mathrm{K}$-addition processes. This is consistent with high $\mathrm{K}_{2} \mathrm{O}$ contents (up to 7.50\%) in most of the shale and arkose samples, in comparison to the PAAS (3.70) and UCC (3.40). In moderate to extremely weathered sedimentary rocks, $\mathrm{K}$-addition is related to $\mathrm{K}$-metasomatism [45] [56]. The trend of $\mathrm{K}_{2} \mathrm{O}$ enriched samples is parallel to A-K join and also suggests that the $\mathrm{K}$-addition has resulted in the conversion of secondary clay mineral such as kaolinite into illite.

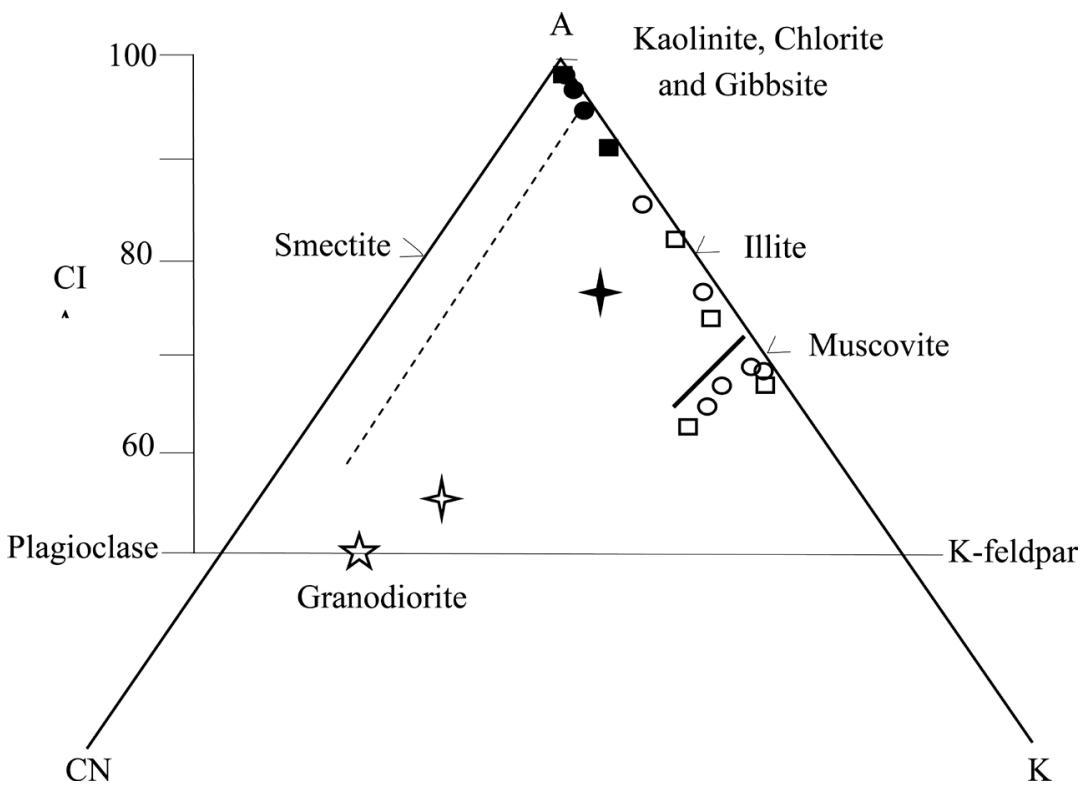

Figure 10. A-CN-K plots [54] for sediment samples from Kumba area. The dotted line represents the possible weathering trend of the source rock if no K-addition is involved. The heavy solid line represents possible weathering trend combined with K-metasomatism from the source rock. The symbols are the same as in Figure 4. 
Since the CIA index is less sensitive to the weathering degree when $\mathrm{K}$ reintroduction occur in the system, as it is probably the case for the present study, the Chemical index of Weathering $\left[\mathrm{CIW}=\left(\mathrm{Al}_{2} \mathrm{O}_{3} /\left(\mathrm{Al}_{2} \mathrm{O}_{3}+\mathrm{CaO}^{*}+\mathrm{Na}_{2} \mathrm{O}\right)\right] \times 100\right.$ (Table 1) which is not sensitive to post-depositional $\mathrm{K}$-enrichment can be used to monitor paleo-weathering at the source [47]. All the samples have CIW values higher than 87, indicative of extreme chemical weathering. This, in turn, accords with data obtained using PIA index.

The $\mathrm{K}_{2} \mathrm{O} / \mathrm{Al}_{2} \mathrm{O}_{3}$ ratios also reflect weathering processes, since they indicate presence of clay minerals. The $\mathrm{K}_{2} \mathrm{O} / \mathrm{Al}_{2} \mathrm{O}_{3}$ ratios for clay minerals and feldspars are different ( 0.0 to 0.3 and 0.3 to 0.9 respectively) [46]. The average $\mathrm{K}_{2} \mathrm{O} / \mathrm{Al}_{2} \mathrm{O}_{3}$ ratio for $\mathrm{Fe}$-shale and shale is 0.25 and is 0.26 for sandstone. These ratios are closer to the clay mineral range in both mudrock and sandstone samples, indicating the leaching of almost all the feldspar in the studied samples. However, enrichment in $\mathrm{K}_{2} \mathrm{O} / \mathrm{Na}_{2} \mathrm{O}$ ratio (1.00 - 403.00) reflects decomposition of plagioclase than K-feldspar during weathering [57] [58] and/or K-reintroduction in the system during diagenesis [59] [60]. The weathering of almost all the plagioclase is attested by the markedly depletion of the elements $\mathrm{Ca}$ and $\mathrm{Sr}$ relative to PAAS and UCC, since these elements are contained in minerals that weather rapidly such as plagioclase [44].

For Th/U in sedimentary rocks, weathering tends to result in oxidation of insoluble $\mathrm{U}^{4+}$ to soluble $\mathrm{U}^{6+}$, with loss of solution and elevation of Th/U ratios [19] [20] [61]. The increase in these ratios can be attributed to intermediate and intense weathering when $\mathrm{Th} / \mathrm{U}$ is greater than 3 and 4, respectively [9] [62]. The $\mathrm{Th} / \mathrm{U}$ ratios of mudrocks and sandstones from Kumba range from 2.29 to 9.32 (Table 2) with only 3 ratios less than 3 and most ratios greater than 4 . Moreover, many of the Th/U ratios (up to 9.32) are far above the PAAS value of 4.71. In this sense, these sediments might have been derived from a source subjected to significant weathering.

Furthermore, intense weathering produces LREE/HREE fractionation [63], although the REE are quantitatively transferred from the source to the sediment [64]. This is possibly due to preferential HREE retention in solution [65]. In this study, LREE/HREE ratios vary between 5.84 and 20.1, indicating remarkable fractionation and suggesting, as noted above, that weathering was intense at that time.

\subsection{Provenance}

Provenance analysis of sediments is aimed at reconstructing the parent-rock assemblages of sediments [66]. $\mathrm{Al}_{2} \mathrm{O}_{3} / \mathrm{TiO}_{2}$ ratio is widely used to identify sediment provenance [67] because $\mathrm{Ti}$ is less affected by weathering [68] [69]. Al also behaves as a relatively conservative element during weathering [70]. In order to identify the source rock, the $\mathrm{Al}_{2} \mathrm{O}_{3} / \mathrm{TiO}_{2}$ ratios have been calculated and the elemental concentration of sediment plotted (Figure $11(\mathrm{a})$ ). The $\mathrm{Al}_{2} \mathrm{O}_{3} / \mathrm{TiO}_{2}$ ratio in terrigenous sediments increases from mafic to felsic rocks, varying between 3 and 8 for mafic igneous rocks, 8 - 21 for intermediate rocks and $21-70$ for felsic rocks [72] [73] [74]. Except one sample, shale and arkose of the studied samples 
exhibit $\mathrm{Al}_{2} \mathrm{O}_{3} / \mathrm{TiO}_{2}$ ratios ranging from 16.03 to 91.67 , suggesting geochemical signature of both intermediate and felsic igneous rock whereas Fe-shale and Fe-sandstone (4.75 - 13.78) indicate contribution of both intermediate and mafic rock. Figure 11 (a) shows the comparison of $\mathrm{Al}_{2} \mathrm{O}_{3}$ vs. $\mathrm{TiO}_{2}$ between the Kumba mudrocks and sandstones and average compositions of UCC and PAAS. Location of the samples in the $\mathrm{TiO}_{2}$ vs. $\mathrm{Zr}$ scatter plot confirm geochemical signature of a provenance comprising dominantly felsic igneous rock with lesser contribution of intermediate rocks (Figure 11(b)).
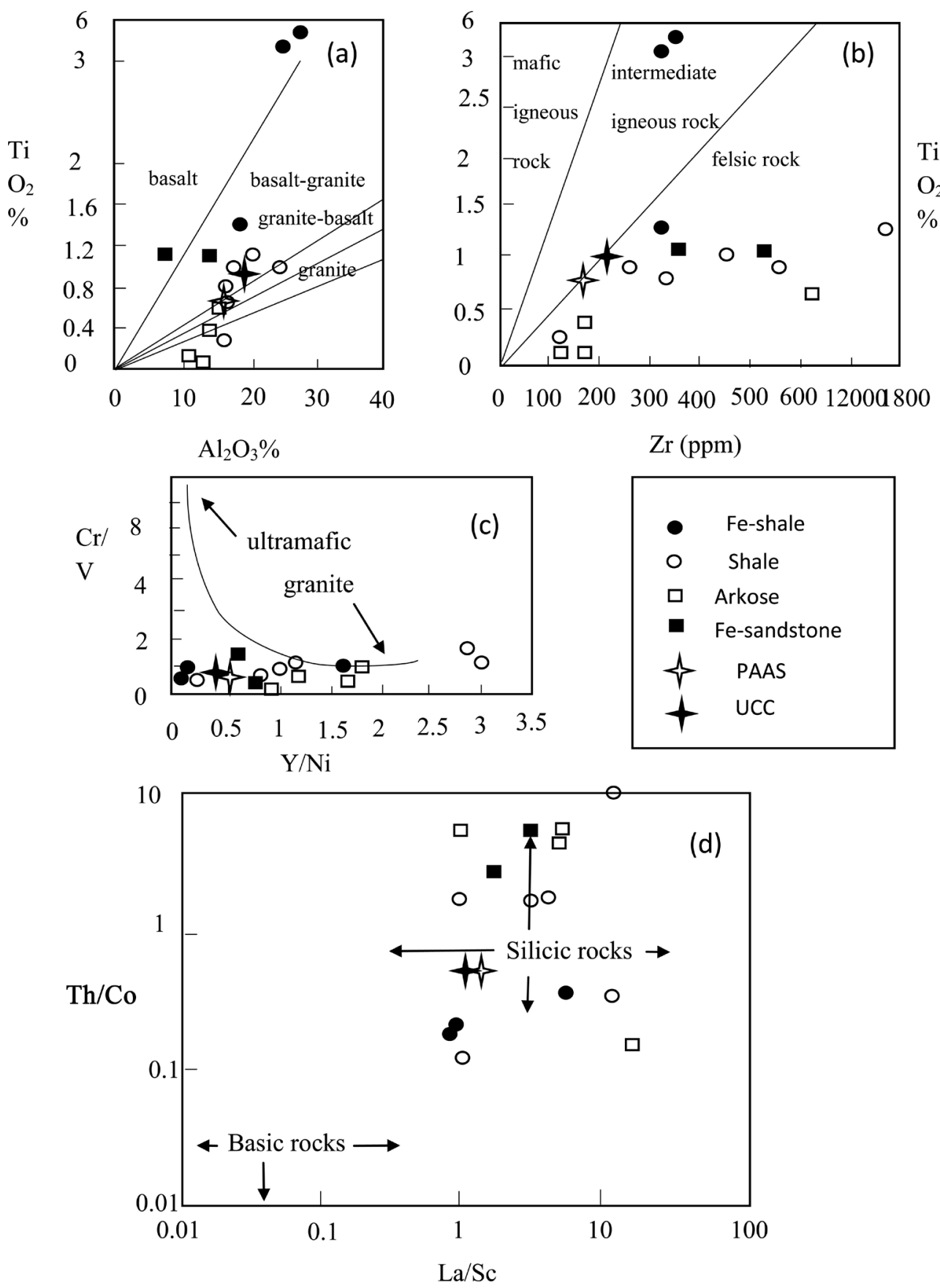

Figure 11. Scatter plots of (a) $\mathrm{Al}_{2} \mathrm{O}_{3}$ vs. $\mathrm{TiO}_{2}$, (b) $\mathrm{Zr}$ vs. $\mathrm{TiO}_{2}$, (c) $\mathrm{Y} / \mathrm{Ni}$ vs. $\mathrm{Cr} / \mathrm{V}$ and (d) $\mathrm{Th} / \mathrm{Co}$ vs. $\mathrm{La} / \mathrm{Sc}[71]$ for the studied sediments. 
Provenance of clastic sediments can also be constrained, as suggested by previous studies, by the concentrations of elements and elemental ratios such as $\mathrm{Cr}$, $\mathrm{Ni}$, and $\mathrm{Cr} / \mathrm{Ni}, \mathrm{Y} / \mathrm{Ni}, \mathrm{Cr} / \mathrm{V}$ respectively [64] [75] [76]. Cr is strongly concentrated in chrome spinel that is a key mineral in ultramafic and mafic rocks. Very high concentrations of the trace elements $\mathrm{Cr}$ (up to $1528 \mathrm{ppm}$ ) [75] and $\mathrm{Ni}$ are indicative of significant contribution from a mafic-ultramafic source component in sedimentary rocks. Furthermore, Garver et al. [76] suggested that values of $\mathrm{Cr}>150$ and $\mathrm{Ni}>100 \mathrm{ppm}$ are diagnostic of mafic-ultramafic rocks in the source area. Compared to the PAAS (110), the relatively higher $\mathrm{Cr}$ concentration $(120-600 \mathrm{ppm})$ of some Fe-shale and Fe-sandstone might be related to contribution from the mafic source rocks. $\mathrm{Cr} / \mathrm{Ni}$ between 1.3 and 1.5 ratios reflect the contribution of mafic-ultramafic rocks whereas higher $\mathrm{Cr} / \mathrm{Ni}$ ratios $>2$ typify an input of mafic volcanic detritus; but it is noteworthy that these ratios $>3$ for sandstones suggest significant sedimentary fractionation [76]. With only a few exceptions, the studied samples have $\mathrm{Cr} / \mathrm{Ni}$ ratios $>2$; these ratios are high (up to 7.62) for sandstone samples, suggesting an input of mafic rock and/or a significant fractionation of the sedimentary rocks of the Kumba area during transport and deposition. The relative contribution from mafic-ultramafic source in comparison to the felsic rocks have been evaluated by plotting the sediments in the $\mathrm{Y} / \mathrm{Ni}$ vs. $\mathrm{Cr} / \mathrm{V}$ scatter plot of [77] (Figure 11(c)). The ultra-mafic rocks are characterized by lower $\mathrm{Y} / \mathrm{Ni}$ and Higher $\mathrm{Cr} / \mathrm{V}$ [64]. Most of the samples have slightly higher $\mathrm{Cr} / \mathrm{V}$ compared to both UCC and PAAS, indicating probably a noticeable contribution of mafic to ultra-mafic rocks. It is noteworthy that the $\mathrm{Cr}$ enrichment could be associated with the fractionation of $\mathrm{Cr}$-bearing mineral in sediments as a result of different sedimentary processes [76]. On the other hand, the Th/Co vs. La/Cs plot [71] suggests that almost all the studied samples were derived from felsic source rocks rather than mafic source rocks (Figure 11(d)). It follows that the interpretation supporting mafic input in the samples studied must be made cautiously.

In addition, the shape of REE patterns and the size of the $\mathrm{Eu}$ anomaly have been also used to infer sources of sedimentary rocks [9] [63] [78]. Since basic igneous rock contain low LREE/HREE ratios and little or no Eu to positive Eu anomalies, whereas silicic igneous rocks usually contain higher LREE/HREE ratios and negative Eu anomaly [79] [80]. LREE/HREE ratios of this study are variable, ranging from 5.84 to 20.91. Some values are lower than the PAAS (13.8) and UCC (9.54) and other higher (up to 20.91), indicating the derivation of sediments from a source area composed of felsic and mafic source rocks. The PAAS-normalized REE pattern shows markedly positive Eu anomalies for average of Fe-shale and shale samples (Figure 7(a)) and the chondrite [49] normalized REE patterns of the studied rocks exhibit slight negative or no Eu anomaly, as shown in Figure $7(\mathrm{~b})$. These features, particularly the positive and negative $\mathrm{Eu}$ anomalies $\left(\mathrm{Eu} / \mathrm{Eu}^{*} \approx 0.87-1.62\right)$, are indicative of provenance including both felsic and mafic nature of protolith. It is important to observe that, it could be expected that $\mathrm{Eu} / \mathrm{Eu}^{*}$ should increase (i.e., a prominent negative $\mathrm{Eu} / \mathrm{Eu}^{*}$ ), as 
more feldspar is destroyed in a second weathering cycle [81]. On this basis we may state that the positive $\mathrm{Eu} / \mathrm{Eu}^{*}$ of some of the studied samples, slightly higher than that of the PAAS, could likely monitor minor contribution of mafic components. The limited importance of a mafic supply is confirmed also by other provenance proxies including the $\mathrm{Cr} / \mathrm{V}$ and $\mathrm{Y} / \mathrm{Ni}$ ratios.

The chondrite normalized REE of the samples studied have LREE and HREE patterns and contents roughly similar to those of various lithologies of expected plutonic source rocks intruding the exposed surrounding migmatitic-gneiss basement, but display different $\mathrm{Eu}$ anomalies. The arkose and Fe-sandstone samples, with slight negative or no anomalies, have REE pattern comparable to that of the MFG (Figure 9(a)). The latter are massive with modal composition of diorite, quartz-diorite, quartz-monzodiorite, quartz monzo-gabbro or compositionally banded with light felsic layers (granodioritic to monzogranitic) alternating with dark mafic layer (dioritic to quartz dioritic) [34]. It could have acted, to a certain extent, as a source of the mafic component of the studied sediments. The LREE pattern and the Eu anomalies of shale underline to a certain extent the signature of CGG (Figure 9(a)). This rock, together with biotite-rich granite, porphyritic granite, alkali feldspar granite and two-mica granite [35] could have served as the felsic protolith for the Kumba sedimentary rocks. However, the prominent negative Eu anomalies of the latter (Figure 9(b)) are inconsistent with the slight negative or no $\mathrm{Eu}$ anomalies of the studied sediments. On the other hand, any significant contribution of these granitoid bodies to the sediments would result in reduced Eu anomalies of the samples. In addition, the lack of significant negative Eu anomalies and overall flat HREE patterns implies a granodiorite rather than a granite source [53] [82]. This would indicate that, although their contribution cannot be excluded, these granitoids are not the main source of the Kumba sediments. The contribution of the extensive Neoproterozoic migmatitic-gneiss basement rocks exposed relatively adjacent to the study area is possible. The fact that the Precambrian rocks [tonalite-tronjhemite-gneiss (TTG), granodiorite and quartz diorite] generally exhibit positive Eu anomalies and high LREE/HREE ratios, as pointed out by Cullers and Graf [83], and Nagarajan et al. [19], further supports this idea. These migmatitic gneisses were metamorphosed under amphibolite-facies conditions and are in many places cross-cut by mafic dykes [35]. These mafic dykes could have acted, to a lesser extent, as the source of mafic materials. However, the chemical analyses for these neighboring migmatitic-gneiss rocks, as well as those of the mafic dykes, are not available.

\subsection{Sorting and Recycling}

It is well known that transport and deposition of clastic sediments involve mechanical sorting. Their effect on the chemical composition of terrigeneous sediments is important and may affect the distribution of paleoweathering and provenance proxies [16] [84]. The distribution of the chemical components winthin a suite is mainly determined by the mechanical properties of the host 
minerals. The process basically fractionates $\mathrm{Al}_{2} \mathrm{O}_{3}$ (clay minerals) from $\mathrm{SiO}_{2}$ (quartz and feldspars). The $\mathrm{SiO}_{2} / \mathrm{Al}_{2} \mathrm{O}_{3}$ ratio reflects the abundance of quartz as well as the feldspar content of the sediments [85] [86]. This ratio can be used to understand both the sorting and recycling of sediments. With increasing sediment recycling, the proportion of quartz among sediments will increase, whereas feldspar and mafic minerals would decrease. As a result, the $\mathrm{SiO}_{2} / \mathrm{Al}_{2} \mathrm{O}_{3}$ ratio would progressively increase [87]. In this study, the $\mathrm{SiO}_{2} / \mathrm{Al}_{2} \mathrm{O}_{3}$ ratio is low in Fe-shale and shale and range from 1.19 to 4.12 with an average of 2.89. Whereas this ratio is relatively higher in arkose and Fe-sandstone and ranges from 4.69 to 9.94 with an average of 6.65. This $\mathrm{SiO}_{2} / \mathrm{Al}_{2} \mathrm{O}_{3}$ ratio is always $>1$ and mostly higher than those of the PAAS (3.32; Table 2) suggesting quartz enrichment in the sediments and thus chemical maturity of the studied rocks.

Sorting also fractionates $\mathrm{TiO}_{2}$, mostly present in clay minerals and Ti-oxides, from $\mathrm{Zr}$, present in zircon, and sorted with quartz. However, the variable content of inert elements in mudrocks is mostly due to the weathering degree, which affects parent rocks. Ternary plots based on $\mathrm{Al}_{2} \mathrm{O}_{3}, \mathrm{TiO}_{2}$ and $\mathrm{Zr}$ eliminate the weathering effects and may illustrate the presence of sorting-related fractionations which are recognizable by simple mixing trends on a ternary $\mathrm{Al}_{2} \mathrm{O}_{3}-\mathrm{TiO}_{2}$ $\mathrm{Zr}$ diagram [88]. In the present study a mixing trend, mostly characterized by changes in $\mathrm{Al}_{2} \mathrm{O}_{3} / \mathrm{Zr}$, which could be due to a recycling effect, is clearly envisaged (Figure 12(a)). This is consistent with the low ICV values $(<1)$ in most samples. $\mathrm{Zr} / \mathrm{Sc}$ ratio is also a useful index of sediment recycling [81]. Higher values of this index indicate zircon accumulation by sediment recycling and sorting. Except for two samples, all the sediments have $\mathrm{Zr} / \mathrm{Sc}$ ratios ranging between 20.17 and 168 , indicating zircon accumulation by sediment recycling and sorting. Zircon, and thus $\mathrm{Zr}$ enrichment during sorting, can also be evaluated when the $\mathrm{Zr} / \mathrm{Sc}$ ratio is plotted against the $\mathrm{Th} / \mathrm{Sc}$ ratio, an indicator of chemical differentiation [64]. The first cycle sediments show a simple positive correlation of $\mathrm{Th} / \mathrm{Sc}$ with $\mathrm{Zr} / \mathrm{Sc}$, whereas the $\mathrm{Zr} / \mathrm{Sc}$ ratio will increase more rapidly than $\mathrm{Th} / \mathrm{Sc}$ with the increasing recycling of sediments [80]. The sedimentary rocks of Kumba area have $\mathrm{Zr} / \mathrm{Sc}$ ratios ranging between 9.78 and 168 accompanied by $\mathrm{Th} / \mathrm{Sc}$ ratios ranging between 0.13 and 6.04 that suggest, according to Mclennan et al. [64] compositional variation from source area. In the scatter plot of $\mathrm{Zr} / \mathrm{Sc} v \mathrm{vs}$. Th/Sc, all the samples are not clustered along the primary compositional trend but fall along a trend involving zircon addition (Figure 12(b)) and thus sediment recycling; this is consistent with $\mathrm{Al}_{2} \mathrm{O}_{3}-\mathrm{TiO}_{2}-\mathrm{Zr}$ diagram.

The $\mathrm{K}_{2} \mathrm{O} / \mathrm{Na}_{2} \mathrm{O}$ ratio is also indicative of sediment recycling. It increases with weathering due to more liable nature of plagioclase relative to K-feldspar [21]. This ratio $>1$ indicates high chemical maturity [89]. The $\mathrm{K}_{2} \mathrm{O} / \mathrm{Na}_{2} \mathrm{O}$ ratios for the studied samples range from 1.00 to 403.00 , with most of them (Table 1) higher than the PAAS (3.08) and UCC (0.87), indicating a high amount of sediment recycling.

In addition, the recycling processes is possibly recorded by the slight similar CIA value (Table 1) for both studied mudrocks (Fe-shales and shales) and sand- 

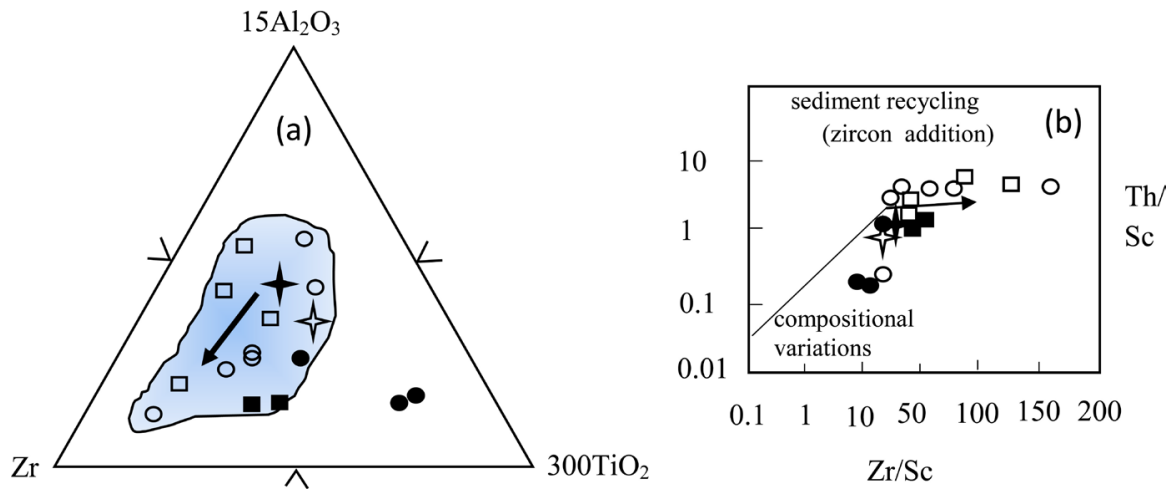

Figure 12. (a) Ternary $15^{*} \mathrm{Al}_{2} \mathrm{O}_{3}-300^{*} \mathrm{TiO}_{2}-\mathrm{Z}$ plot showing possible sorting effects [88] and (b) $\mathrm{Th} / \mathrm{Sc}$ vs. $\mathrm{Zr} / \mathrm{Sc}$ plot of the Kumba sediments. The symbols are the same as in Figure 11.

stones (arkose and Fe-sandstone). Since it is well known that finer grain rocks such as shales should show stronger evidences of weathering than sandstones [90]. These recycling processes might have homogenized, to some extent, compositions. Moreover, very high values of CIA, if the sediments have experienced K-enrichment, may reflect a sediment recycling nature, and thus a cumulative effect [91] [92] [93]. Some of the CIA values for the studied samples, as shown on Table 1, are very high (up to 99.5), indicating that the sediments underwent a recycling process.

Furthermore, the $\Sigma$ REE abundances (Table 3) reflect the recycling process. High $\Sigma$ REE contents suggest a possible control by differing amounts of accessory minerals (e.g., zircon) and/or quartz due to recycling processes [94]. In this study, $\Sigma$ REE values range from 81.17 to $738.75 \mathrm{ppm}$, with an average of 282 ppm, higher than that of the PAAS (184.77 ppm) and UCC (146.37 ppm), suggesting the recycling of the Kumba sediments. It is also important to note that the fractionated REE pattern with slight elevated total light REE ( $L$ LREE) content of mudrocks, ranging from 196.8 to 692.8 (average 343.03), suggests that the sorting or recycling is slight higher for the mudrocks than the sandstones, with LLREE ranging between 75.13 and 211.6, average100.39. It follows that, although harmonization of the mudrock and sandstone can be envisaged, as discussed earlier, REE geochemistry of these samples is still, to a lesser extent, different.

\subsection{Paleo-Oxidation Conditions}

$\mathrm{Ni} / \mathrm{Co}$ and $\mathrm{V} / \mathrm{Cr}$ ratios are sensitive to the paleoredox conditions of ancient sediments [19] [95] [96]. Sedimentary rocks derived from oxic conditions are characterized by $\mathrm{Ni} /$ Co ratios below 5 ; between 5 and 7 , these ratios are related to dysoxic conditions whereas values greater than 7 reflect suboxic to anoxic conditions [95]. $\mathrm{V} / \mathrm{Cr}$ ratios $<2$, ranging between 2 and 45.25 and $>4.25$ indicate oxic, dysoxic and suboxic to anoxic conditions respectively. Except for 3 samples, the studied sediments show $\mathrm{Ni} / \mathrm{Co}$ ratio always less than 7 , reflecting oxic and dysoxic conditions. Whereas all the samples exhibit $\mathrm{V} / \mathrm{Cr}$ ratios less than 2, suggesting oxic environment. Other ratios such as $\mathrm{U} / \mathrm{Th}$ and $\mathrm{Cu} / \mathrm{Zn}$ are also useful 
in determining redox conditions of clastic rocks [19] [61] [97]. Oxic conditions of deposition are characterized by $\mathrm{U} / \mathrm{Th}$ ratios below 1.5 whereas values above 1.25 indicate suboxic and anoxic conditions [98]. High $\mathrm{Cu} / \mathrm{Zn}$ values suggest reducing depositional conditions, whereas low $\mathrm{Cu} / \mathrm{Zn}$ values are related to oxidizing environments. Except one sample, all the U/Th ratios of the studied sediments are less than 1.25 , supporting oxidizing conditions. $\mathrm{Cu} / \mathrm{Zn}$ ratios, in the present samples, range from 0.01 to 1.2 , with most of the data less than 1 , revealing the depositional environment of these samples to be oxic. Ce anomaly can also help to highlight the paleoredox conditions of terrigenous sediments. Since $\mathrm{Ce}$ is easily oxidized to $\mathrm{Ce}^{+4}$ in oxidizing conditions and therefore remain in sediments, causing a positive $\mathrm{Ce}$ anomaly with $\mathrm{Ce} / \mathrm{Ce}^{\star}$ ratios $>0.10$. Whereas, an anoxic environment will cause a negative $\mathrm{Ce}$ anomaly with $\mathrm{Ce} / \mathrm{Ce}^{*}$ ratio < 0.10 [46] [99]. For the current study, the Ce anomaly ranged from 0.88 to 1.03 , which is greater than 0.10 , supporting an oxic condition. Taken collectively, $\mathrm{Ni} / \mathrm{Co}$ and $\mathrm{V} / \mathrm{Cr}$ ratios and $\mathrm{Ce}$ anomaly clearly indicate that the depositional conditions for the studied sediments were oxic to suboxic. This result is in agreement with a depositional milieu ranging from a restricted to full marine environment, which are supposed to be broadly suboxic to oxic respectively [61] [98]. This is the case for the studied sediments which were deposited in such an environment during the post-rift (Drift I) stage of the tectonic history of the Douala sub-basin, related to the opening of the South Atlantic Ocean [3] [33] [36] [37] (Figure 2).

\subsection{Tectonic Setting}

Plate tectonic settings of the provenance of clastic sedimentary rocks control the chemical composition of these rocks. To constraint the tectonic settings of ancient sedimentary basins from geochemical characteristics, it is presumed that the nature of the source terrain is intimately related to processes controlling the origin and evolution of adjacent lying sedimentary basin [11] [28]. Major elements of clastic sedimentary rocks, such as the $\mathrm{SiO}_{2}, \mathrm{~K}_{2} \mathrm{O}$ and $\mathrm{Na}_{2} \mathrm{O}$, are valuable in determining the tectonic setting from terrigenous sedimentary rocks. $\mathrm{SiO}_{2}$ and $\mathrm{K}_{2} \mathrm{O} / \mathrm{Na}_{2} \mathrm{O}$ increase from volcanic-arc to active continental margin to passive margin setting [12] [100]. In the $\mathrm{SiO}_{2}-\mathrm{K}_{2} \mathrm{O} / \mathrm{Na}_{2} \mathrm{O}$ discrimination diagram of Roser and Korsch [12], most samples fall in the general area of active continental margin field, except for a few in the oceanic island arc margin (Figure 13(a)).

The Th-Sc-Zr/10 ternary diagram of Bhatia and Crook [11] has also been used to differentiate the tectonic setting of the studied sediments. This diagram is believed to be most useful trace element tectonic discrimination plots. Samples from Kumba sediments are relatively scattered on Th-Sc-Zr/10 ternary diagram with data spreading across three fields: the passive continental margin (PM), the continental island arc (CIA) and the active continental margin (ACM), suggesting that the tectonic setting of the Kumba sediments may be more complex (Figure 13(b)).

This complexity, most likely resulting from an inherited signature, is probably 

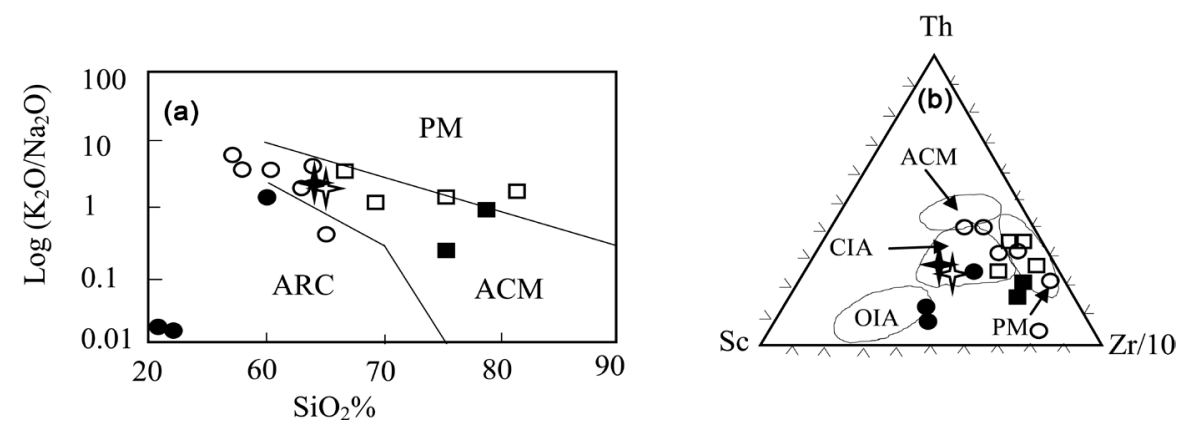

Figure 13. Discrimination diagrams to understand the different tectonic settings of the studied rocks. (a) Scatter plot of $\mathrm{SiO}_{2}$ vs. $\mathrm{K}_{2} \mathrm{O} / \mathrm{Na}_{2} \mathrm{O}$ [12] and (b) triangular plot of Th-Sc- $\mathrm{Zr} / 10$ [11], $\mathrm{PM}=$ passive margin, $\mathrm{ACM}=$ active continental margin, $\mathrm{ARC}=$ oceanic island arc margin, OIA = continental island arc. The symbols are the same as in Figure 11.

related to recycling effect experienced by the samples studied, since some sediments can be transported from their tectonic setting of origin into a sedimentary basin in a different tectonic environment [90]. Such discrepancy may also reflect a sediment derived from potentially multiple sources (i.e., mixed provenance) [26] [94]. In this study, the result obtained can be partially explained by the possible contribution, in the Kumba sediments, deposited in a less tectonically drift-period, of reworked materials from the underlying Lower Mundeck Formation, characterizing the rift-drift and still tectonically active period. Similarly, it is significant to note that the Cretaceous Lower Mundeck Formation overlies unconformably the Pan-African Precambrian basement, exposed relatively adjacent to the study area (Figure 1) and have experienced the contribution of reworked materials of this Precambrian [3] [33] [36] [37] and probably of the unknown Paleozoic deposits, which have certainly been thoroughly eroded and thus have never been documented [37]. Therefore, besides the possible contribution of the adjacent Precambrian basement, the recycling of preexisting Lower Cretaceous and probable Paleozoic clastic rocks could explain the complex compositional signal of the Kumba sediments.

\section{Conclusions}

In this study, fifteen Cretaceous sediment samples from Kumba area in the Douala sub-basin were analyzed in order to determine the provenance, source rock weathering, paleo-oxidation conditions of the depositional environment and tectonic setting of these rocks. Based on results and discussion, the following conclusions are reached:

1) The studied rocks have been classified, on the basis of their major element composition, mainly as Fe-shale, shale, arkose and Fe-sandstone.

2) These rocks were mainly derived from felsic igneous rocks with lesser contribution of mafic component as suggested by the plot of $\mathrm{Zr}$ vs. $\mathrm{TiO}_{2}, \mathrm{Y} / \mathrm{Ni}$ vs. $\mathrm{Cr} / \mathrm{V}, \mathrm{TiO}_{2}$ vs. $\mathrm{Al}_{2} \mathrm{O}_{3}$ diagram, $\mathrm{REE}$ characteristics and Eu anomalies.

3) The PIA and CIW values suggest that the sediments and their potential source rocks were subjected to intense weathering. Whereas the CIA and $\mathrm{K}_{2} \mathrm{O}$ / $\mathrm{Na}_{2} \mathrm{O}$ ratio in most samples could be indicative of $\mathrm{K}$-addition processes. The 
A-CN-K plot also suggests a possible potash metasomatism during diagenesis.

4) The Ce anomalies and the values of $\mathrm{Ni} / \mathrm{Co}, \mathrm{U} / \mathrm{Th}$ and $\mathrm{Cu} / \mathrm{Zn}$ show that the redox condition during the sedimentation were suboxic to oxic.

5) The $\mathrm{SiO}_{2}-\mathrm{K}_{2} \mathrm{O} / \mathrm{Na}_{2} \mathrm{O}$ and $\mathrm{Th}-\mathrm{Sc}-\mathrm{Zr} / 10$ tectonic discrimination diagrams show that the studied rocks were deposited mainly in an active continental margin setting, but also in a passive margin and various tectonic environments, reflecting probably the recycling effect experienced by the samples studied.

6) The geochemical compositional trends of sedimentary rocks from the Upper Mundeck Formation, outcropping at the Kumba area, document the paleogeography of the Douala sub-basin. These rocks are, in some extent, material embodiments of the beginning of the separation of South American and African plates during Early Cretaceous.

\section{Acknowledgements}

The authors express their gratitude to the ALS Geochemical Laboratory (Galway, Ireland) for geochemical analyses. They are also grateful to the anonymous reviewers for their constructive reviews and comments. This research did not receive any specific grant from funding agencies in the public, commercial, or not-for-profit sector.

\section{Conflict of Interests}

The authors do not have any conflict of interests.

\section{References}

[1] Pauken, R.J. (1992) Sanaga Sud Field, Offshore Cameroon, West Africa. In: Halbouty, M.T., Ed., Giant Oil and Gas Fields of the Decade 1978-1988, the American Association of Petroleum Geologists Memoir, 54, 217-230.

[2] Pauken, R.J., Thompson, J.M., Schuman, J.R. and Cooke, J.C. (1991) Geology of the Douala Basin, Offshore Cameroon. The American Association of Petroleum Geologists Bulletin, 75, 651-652.

[3] Nguene, F.R., Tamfu, S.F., Loule, J.P. and Ngassa, C. (1992) Palaeoenvironments of the Douala and Kribi/Campo Sub-Basins, in Cameroon, West Africa. In: Curnelle, R., Ed., Géologie Africaine, 1 er Colloque de Stratigraphie et de Paléogéographie des Bassins Sédimentaires Ouest-Africains, 2ème Colloque Africain de Micropaléontologie, Libreville, Gabon, 1991, Recueil des Communications: Boussens, Elf Aquitaine, 129-139.

[4] Ackerman, W.C., Boatwright, D.C., Burwood, B.M., Van Lerberche, D., Bondjo, E., Tamfu, S.F. and Ovono, D. (1993) Geochemical Analysis of Selected Hydrocarbon Samples in the Douala Basin, Cameroon. Implications for an Oil-Prone Source Rock. The American Association of Petroleum Geologists Bulletin, 77, 1604.

[5] Tamfu, S.F., Batupe, M., Pauken, R.J. and Boatwright, D.C. (1995) Geological Setting, Stratigraphy and Hydrocarbon Habitat of the Douala Basin, Cameroon. The American Association of Petroleum Geologists Bulletin, 79, 95.

[6] Brownfield, M.E. and Carpenter, R.R. (2006) Geology and Total Petroleum Systems of the West-Central Coastal Province (7203), West Africa. US Geological Survey Bulletin, 2207-B.

[7] Ndonwie Mahbou, E. (2007) Petrophysical Characterization of Petroleum Reser- 
voirs and Source Beds of the Batanga Sud-Block, Kribi-Campo Sub-Basin, Cameroon. AAPG Annual Convention, Abstract.

[8] Njiké Ngaha, P.R. (1984) Contribution à l'Etude Géologique, Stratigraphique et Structurale de la Bordure du Bassin Atlantique du Cameroun. Thèse 3ème Cycle, Université de Yaoundé, Cameroun.

[9] Taylor, S.R. and McLennan, S.M. (1985) The Continental Crust: Its Composition and Evolution. Blackwell, Oxford.

[10] Bhatia, M.R. (1983) Plate Tectonics and Geochemical Composition of Sandstone. The Journal of Geology, 91, 611-627. https://doi.org/10.1086/628815

[11] Bhatia, M.R. and Crook, K.A.W. (1986) Trace Element Characteristics of Graywackes and Tectonic Setting Discrimination of Sedimentary Basins. Contributions to Mineralogy and Petrology, 92, 181-193. https://doi.org/10.1007/BF00375292

[12] Roser, B.P. and Korsch, R.J. (1986) Determination of Tectonic Setting of SandstoneMudstone Suites Using $\mathrm{Si}_{2} \mathrm{O}$ Content and $\mathrm{K}_{2} \mathrm{O} / \mathrm{Na}_{2} \mathrm{O}$ Ratio. The Journal of Geology, 94, 635-650. https://doi.org/10.1086/629071

[13] Roser, B.P. and Korsch, R.J. (1988) Provenance Signatures of Sandstone-Mudstone Suites Determined Using Discriminant Function Analysis of Major-Element Data. Chemical Geology, 67, 119-139.

[14] Condie, K.C., Philip, D.N.J. and Conway, C.M. (1992) Geochemical and Detrital Mode Evidence for two Sources of Early Proterozoic. Sedimentary Geology, 77, 5176.

[15] Condie, K.C., Lee, D. and Farmer, G.L. (2001) Tectonic Setting and Provenance of the Neoproterozoic Uinta Mountain and Big Vottonwood Groups, Northern Utah: Constraints from Geochemistry, Nd Isotopes, and Detrital Modes. Sedimentary Geology, 141-142, 443-464.

[16] Bauluz, B., Mayayo, M.J., Fernandez-Nieto, C. and Gonzalez Lopez, J.M. (2000) Geochemistry of Precambrian and Paleozoic Siliciclastic Rocks from the Iberian Range (NE Spain): Implications for Source-Area Weathering, Sorting, Provenance, and Tectonic Setting. Chemical Geology, 168, 135-150.

[17] Bhat, M.J. and Ghosh S.K. (2001) Geochemistry of the 2.51 Ga Old Rampur Group Pelite, Western Himalayas: Implications for their Provenance and Weathering. Precambrian Research, 108, 1-16.

[18] Dayal, A.M. and Murthy, S.N. (2006) Geochemistry of Pakhal Shales. Journal of the Geological Society of India, 67, 770-776.

[19] Nagarajan, R., Madhavaraju, J., Nagendra, R., Armstrong-Altrin, J.S. and Moutte J. (2007) Geochemistry of Neoproterozoic Shales of Rabanpalli Formation, Bhima Basin, Northern Karnataka, Southern India: Implications for Provenance and $\mathrm{Pa}$ leo-Redox Conditions. Revista Mexicana de Ciencias Geológicas, 24, 150-160.

[20] Nagarajan, R., Armstrong-Altrin, J.S., Nagendra, R., Madhavaraju, J. and Moutte, J. (2007) Petrography and Geochemistry of Terrigenous Sedimentary Rocks in the Neoproterozoic Rabanpalli Formation, Bhima Basin, Northern Karnataka, Southern India: Implications for Paleoweathering Condition, Provenance, and Source Rocks Composition. Journal of the Geological Society of India, 70, 297-312.

[21] Nesbitt, H.W. and Young G.M. (1982) Early Proterozoic Climates and Plate Motions Inferred from Major Element Chemistry of Lutites. Nature, 299, 715-717. https://doi.org/10.1038/299715a0

[22] Mongelli, G. (2004) Rare-Earth Elements in Oligo-Miocenic Pelitic Sediments from Lagonegro Basin, Southern Apennines, Italy: Implications for Provenance and Source-Area Weathering. International Journal of Earth Sciences, 93, 612-620. 
https://doi.org/10.1007/s00531-004-0401-Z

[23] Saxena, A. and Pandit, M.K. (2012) Geochemistry of Hindoli Group Metasediments, SE Aravalli Craton, NW India: Implications for Paleoweathering and Provenance. Journal of the Geological Society of India, 79, 267-278. https://doi.org/10.1007/s12594-012-0045-8

[24] Zaghloul, M.N., Critelli, S., Perri, F., Mongelli, G., Perronne, V., Sonnino, M., Tucker, M., Aiello, M. and Ventimiglia, C. (2010) Depositional Systems, Composition and Geochemistry of Triassic Rifted Continental Margin Redbeds of Internal Rift Chain, Morocco. Sedimentology, 57, 312-350. https://doi.org/10.1111/j.1365-3091.2009.01080.x

[25] Caracciolo, L., Le Pera, E., Muto, F. and Perri, F. (2011) Sandstone Petrology and Musdstone Geochemistry of the Peruc-Korycany Formation (Bohemian Cretaceous Basin, Czech Republic). International Geology Review, 53, 1003-1031. https://doi.org/10.1080/00206810903429011

[26] Concepcion, R.A.B., Dimalanta, C.B., Yumul, G.P., Faustino-Eslava, D.V., Queaño, K.L., Tamayo, R.A. and Imai, A. (2012) Petrography, Geochemistry, and Tectonics of a Rifted Fragment of Mainland Asia: Evidence from the Lasala Formation, Mindoro Island, Philippines. International Journal of Earth Sciences, 101, 273-290. https://doi.org/10.1007/s00531-011-0643-5

[27] Sun, L., Gui, H. and Chen, S. (2012) Geochemistry of Sandstones from the Neoproterozoic Shijia Formation, Northern Anhui Province, China: Implications for Provenance, Weathering and Tectonic Setting. Chemie der Erde-Geochemistry, 72, $253-$ 260.

[28] Akhtar, R.M. (2015) Rare Earth Element Geochemistry of Post- to Neo-Archean Shales from Singhbhum Mobile Belt, Eastern India: Implications for Tectonic Setting and Paleo-Oxidation Conditions. Chinese Journal of Geochemistry, 34, 401409. https://doi.org/10.1007/s11631-015-0052-8

[29] Hourcq, V. (1955) Les Recherches Françaises de Pétrole en Afrique Noire (Cameroun, Afrique Equatoriale Française, Madagascar). I.F.P., Centre d'Etudes Supérieures de Prospection et d'Exploitation des Gisements.

[30] Dumort, J.C. (1968) Carte Géologique du Cameroun à l'Echelle 1/500 000, Feuille de Douala Ouest + Notice Explicative. Direction des Mines et de la Géologie.

[31] Salard-Chebodaeff, M. (1981) Palynologie Maestrichtienne et Tertiaire du Cameroun. Résultats Botaniques. Review of Palaeobotany and Palynology, 32, 401-439.

[32] Meyers, J.B., Rosendahl, B.R., Groschel-Becker, H., Austin, J.J.A. and Rona, P.A. (1996) Deep Penetrating MCS Imaging of the Rift-to-Drift Transition, Offshore Douala and North Gabon Basins, West Africa. Marine and Petroleum Geology, 13, 791-835.

[33] Ntamak-Nida, M.J., Bourquin, S., Makong, J.C., Baudin, F., Mpesse, J.E., Itjoko Ngouem, C., Komguem, P.B. and Abolo, G.M. (2010) Sedimentology and Sequence Stratigraphy from Outcrops of Kribi-Campo Sub-Basin: Lower Mundeck Formation (Lower Cretaceous, Southern Cameroon). Journal of African Earth Sciences, 58, 1-18.

[34] Tagne-Kamga, G. (2003) Petrogenesis of the Neoproterozoic Ngondo Plutonic Complex (Cameroon, West Central Africa): A Case of Late-Collisional Ferro-Potassic Magmatism. Journal of African Earth Sciences, 36, 149-171.

[35] Mosoh Bambi, C.K., Frimmel, H.E., Zeh, A. and Suh, C.E. (2013) Age and Origin of Pan-African Granites and Associated U-Mo Mineralization at Ekomédion, Southwestern Cameroon. Journal of African Earth Sciences, 88, 15-37.

[36] Lawrence, S.R., Munday, S. and Bray, R. (2002) Regional Geology and Geophysics of the Eastern Gulf of Guinea (Niger Delta to Rio Muni). The Leading Edge, 21, 
1117. https://doi.org/10.1190/1.1523752

[37] Regnoult, R.A. (1986) Synthèse Géologique du Cameroun. Direction des Mines et de la Géologie, Yaoundé, Cameroun.

[38] Loule, J.P., Angoua Bioulele, S.E., Seme Abomo, R. and Tamfu, S. (1997) Timing of the Communication between the South and Central Atlantic Using Palynological Data from Eight Wells in the Douala/Kribi-Campo Basin, Cameroon. Second Annual Conference IGCP Project 381, South Atlantic Mesozoic Correlations, Yaoundé, Cameroon, 8-13 March 1997, Abstract.

[39] Batupe, M. and Abolo, M.G. (1997) Guide Book Field Trip N. 2. Mesozoic Syn-Rift Deposits of the Campo Region in the Douala/Kribi-Campo Basin. 13th Colloquium of African Micropaleontology, 3 rd Colloquium on the Stratigraphy and Paleontology of the South Atlantic, 2nd Annual Meeting of the IGCP Project No. 381.

[40] Benkhelil, J., Giresse, P., Poumot, C. and Ngueutchoua, G. (2002) Lithostratigraphic, Geophysical and Morpho-Tectonic Studies of the South Cameroon Shelf. Marine and Petroleum Geology, 19, 499-517.

[41] Samuel, J., Rouault, R. and Besnus, Y. (1985) Analyse Multiélémentaire Standardisée des Matériaux Géologiques en Spectrometrie d'Emission par Plasma à Couplage Indutif. Analusis, 13, 312-317.

[42] Jenner, G.J., Longerich, H.P. and Jackson, S.E. (1990) ICP-MS a Powerful Tool for High Precision Trace Element Analysis in Earth Sciences: Evidence from Analysis of Selected U.S.G.S. Reference Samples. Chemical Geology, 83, 133-148.

[43] Herron, M.M. (1988) Geochemical Classification of Terrigenous Sands and Shales from Core or Log Data. Journal of Sedimentary Petrology, 58, 820-829.

[44] Roddaz, M., Viers, J., Brusset, S., Baby, P., Boucayrand, C. and Hérrail, G. (2006) Controls on Weathering and Provenance in Amazonian Foreland Basin: Insights from Major and Trace Element Geochemistry of Neogene Amazonian Sediments. Chemical Geology, 226, 31-65.

[45] Fedo, C.M., Nesbitt, H.W. and Young, G.M. (1995) Unravelling the Effects of Potassium Metasomatism in Sedimentary Rocks and Paleosols, with Implications for Paleoweathering Conditions and Provenance. Geology, 23, 921-924. https://doi.org/10.1130/0091-7613(1995)023<0921:UTEOPM>2.3.CO;2

[46] Cox, R., Low, D.R. and Culler, R.L. (1995) The Influence of Sediment Recycling and Basement Composition on Evolution of Mudrock Chemistry in the Southwestern United States. Geochimimica et Cosmochimica Acta, 59, 219-2940.

[47] Harnois, L. (1988) The CIW Index: A New Chemical Index of Weathering. Sedimentary Geology, 55, 319-322.

[48] McLennan, S.M. (2001) Relationships between the Trace Element Compositions of Sedimentary Rocks and Upper Continental Crust. Geochemistry, Geophysics, Geosystems, 2, 86-98. https://doi.org/10.1029/2000gc000109

[49] McDonough, W.F. and Sun, S.S. (1995) The Composition of the Earth. Chemical Geology, 120, 223-253.

[50] Amendola, U., Perri F., Critelli, S., Monaco, P., Cirilli, S., Trecci, T. and Rettori, R. (2016) Composition and Provenance of the Macigno Formation (Late OligoceneEarly Miocene) in the Trasimeno Lake Area (Northern Apennines). Marine and Petroleum Geology, 69, 146-167.

[51] Fedo, C.M., Eriksson, K.A. and Krogstad, E.J. (1996) Geochemistry of Shales from the Archean $(3.0 \mathrm{Ga})$ Buhwa Greenstone Belt, Zimbabwe: Implications for Provenance and Source Area Weathering. Geochimimica et Cosmochimica Acta, 60, 1751-1763.

[52] Selvaraj, K. and Chen, C.T.A. (2006) Moderate Chemical Weathering of Subtropical 
Taiwan: Constraints from Solid-Phase Geochemistry of Sediments and Sedimentary Rocks. The Journal of Geology, 114, 101-116. https://doi.org/10.1086/498102

[53] Cullers, R.L. and Podkovyrov, V.N. (2000) Geochemistry of the Mesoproterozoic Lakhanda Shales in Southeastern Yakutia, Russia: Implications for Mineralogical and Provenance Control, and Recycling. Precambrian Research, 104, 77-93.

[54] Nesbitt, H.W. and Young, G.M. (1984) Prediction of Some Weathering Trend of Plutonic and Volcanic Rocks Based on Thermodynamic and Kinetic Consideration. Geochimica et Cosmochimica Acta, 48, 1523-1534.

[55] Fedo, C.M., Young, G.M., Nesbitt, H.W. and Hanchar, J.M. (1997) Potassic and Sodic Metasomatism in the Southern Province of the Canadian Shield: Evidence from the Paleoproterozoic Serpent Formation, Huronian Supergroup, Canada. Precambrian Research, 84, 17-36.

[56] Rainbird, R.H., Nesbitt, H.W. and Donaldson, J.A. (1990) Formation and Diagenesis of a Sub-Huronian Saprolith: Comparison with a Modern Weathering Profile. The Journal of Geology, 98, 801-822. https://doi.org/10.1086/629455

[57] Gu, X.X., Liu, J.M., Zheng, M.H., Tang, J.X. and Qi, L. (2002) Provenance and Tectonic Setting of the Proterozoic Turbidites in Hunan, South China: Geochemical Evidence. Journal of Sedimentary Research, 72, 393-407. https://doi.org/10.1306/081601720393

[58] Armstrong-Altrin, J.S., Nagarajan, R., Balaram, V. and Natalhy-Pineda, O. (2015) Petrography and Geochemistry of Sand from the Chachalacas and Veracruz Beach Areas, Western Gulf of Mexico, Mexico: Constraints on Provenance and Tectonic Setting. Journal of South American Earth Sciences, 64, 199-216.

[59] Madhavaraju, J. and Lee, Y.I. (2010) Influence of Deccan Volcanism in the Sedimentary Rocks of Maastrichtian-Danian Age of Cauvery Basin Southeastern India: Constraints from Geochemistry. Current Science India, 98, 528-537.

[60] Madhavaraju, J. (2015) Geochemistry of Late Cretaceous Sedimentary Rocks of the Cauvery Basin, South India: Constraints on Paleoweathering, Provenance, and End Cretaceous Environment. Chemostratigraphy, 185-214.

[61] Somayajulu, B.L.K., Yadav, D.N. and Sarin, M.M. (1994) Recent Sedimentary Records from the Arabian Sea. Proceedings of the Indian Academy of Sciences, 103, 315327.

[62] Vosoughi Moradi, A., Sari, A. and Akkaya, P. (2016) Geochemistry of the Miocene Oil Shale (Hançilli Formation) in the Çankiri-Çorum Basin, Central Turkey: Implications for Paleoclimate Conditions, Source-Area Weathering, Provenance and Tectonic Setting. Sedimentary Geology, 78, 136-150.

[63] Mongelli, G., Cullers, R., Dinelli, E. and Rottura, A. (1998) Elemental Mobility during the Weathering of Exposed Lower Crust: The Kinzigitic Paragneisses from the Serre, Calabria, Southern Italy. Terra Nova, 10, 190-195. https://doi.org/10.1046/j.1365-3121.1998.104188.x

[64] McLennan, S.M., Hemming, S., McDaniel, D.K. and Hanson, G.N. (1993) Geochemical Approaches to Sedimentation, Provenance and Tectonics. Geological Society of America, Special Paper, 284, 21-40. https://doi.org/10.1130/SPE284-p21

[65] Cantrell, K.J. and Byrne, R.H. (1987) Rare-Earth Element Complexation by Carbonate and Oxalate Ions. Geochimimica et Cosmochimica Acta, 51, 597-605.

[66] Weltje, G.J. and Von Eynatten, H. (2004) Quantitative Provenance Analysis of Sediments: Review and Outlook. Sedimentary Geology, 171, 1-11.

[67] Sheldon, N.D. and Tabor, N.J. (2009) Quantitative Paleoenvironmental and Paleoclimatic Reconstruction Using Paleosols. Earth-Science Reviews, 95, 1-52. 
[68] Wen, Q. (1989) Geochemistry of the Chinese Loess. Science Press, Beijing, 1-285.

[69] Qiao, Y., Hao, Q., Peng, S., Wang, Y., Li, J. and Liu, Z. (2001) Geochemical Characteristics of the Eolian Deposits in Southern China, and their Implications for Provenance and Weathering Intensity. Palaeogeography, Palaeoclimatology, Palaeoeco$\log$, 308, 513-523.

[70] Broecker, W.S. and Peng, T.H. (1982) Tracers in the Sea. Eldigio Press, New York, 1-690.

[71] Cullers, R.L. (2002) Implications of Elemental Concentrations for Provenance, Redox Conditions, and Metamorphic Studies of Shales and Limestones near Pueblo, CO, USA. Chemical Geology, 191, 305-327.

[72] Girty, G.H., Ridge, D.I., Knaack, C., Johnson, D. and Al-Riyami, R.K. (1996) Provenance and Depositional Setting of Paleozoic Chert and Argillite, Sierra Nevada, California. Journal of Sedimentary Research, 66, 107-118.

[73] Hayashi, K.I., Fujisawa, H., Holland, H.D. and Ohmoto, H. (1997) Geochemistry of $1.9 \mathrm{Ga}$ Sedimentary Rocks from Northeastern Labrador, Canada. Geochimimica et Cosmochimica Acta, 61, 4115-4137.

[74] Nagarajan, R., Armstrong-Altrin, J.S., Kessler, F.L., Hidalgo-Moral, E.L., DodgeWan, D. and Taib, N.I. (2015) Provenance and Tectonic Setting of Miocene Siliciclastic Sediments, Sibuti Formation, Northwestern Borneo. Arabian Journal of Geosciences, 8, 8549-8565. https://doi.org/10.1007/s12517-015-1833-4

[75] Wronkiewicz, D.J. and Condie, K.C. (1987) Geochemistry of Archean Shales from the Witwatersrand Supergroup, South Africa: Source-Area Weathering and Provenance. Geochimimica et Cosmochimica Acta, 51, 2401-2416.

[76] Garver, J.I., Royce, P.R. and Smick, T.A. (1996) Chromium and Nickel in Shale of the Taconic Foreland: A Case Study for the Provenance of Fine-Grained Sediments with an Ultramafic Source. Journal of Sedimentary Research, 100, 100-106.

[77] Hiscott, R.N. (1984) Ophiolitic Source Rocks for Taconic-Age Flysch: Trace Element Evidence. Geological Society of America Bulletin, 95, 1261-1267. https://doi.org/10.1130/0016-7606(1984)95<1261:OSRFTF>2.0.CO;2

[78] Cullers, R.L. (2000) The Geochemistry of Shales, Siltstones and Sandstones of Pennsylvanian-Permian Age, Colorado, USA: Implications for Provenance and Metamorphic Studies. Lithos, 51, 181-203.

[79] Cullers, R.L. (1994) The Controls on the Major and Trace Element Variation of Shales, Siltstones, and Sandstones of Pennsylvanian-Permian Age from Uplifted Continental Blocks in Colorado to Platform Sediment in Kansas, USA. Geochimimica et Cosmochimica Acta, 58, 4955-4972.

[80] El-Bialy, M. (2013) Geochemistry of the Neoproterozoic Metasediments of Malhaq and Um Zarig Formations, Kid Metamorphic Complex, Sinai, Egypt: Implications for Source-Area Weathering, Provenance, Recycling, and Depositional Tectonic Setting. Lithos, 175, 68-85.

[81] Hassan, S., Ishiga, H., Roser, B.P., Dozen, K. and Naka, T. (1999) Geochemistry of Permian Triassic Shales in the Salt Range, Pakistan: Implications for Provenance and Tectonism at the Gondwana Margin. Chemical Geology, 168, 293-314.

[82] Condie, K.C. (1993) Chemical Composition and Evolution of the Upper Continental Crust: Contrasting Results from Surface Samples and Shales. Chemical Geology, 104, 1-37.

[83] Cullers, R.L. and Graf, J. (1984) Rare Earth Element in Igneous Rocks of the Continental Crust: Intermediate and Silicic Rocks, Ore Petrogenesis. In: Henderson, P., Ed., Rare Earth Geochemistry, Elsevier, Amsterdam, 275-316. 
[84] Le Pera, E., Mongelli, G. and Critelli, S. (2000) Compositional and Geochemical Signature of Provenance in Modern Sediments from the Tyrrhenian Continental Shelf, Calabria, Italy. Giornale di Geologia, 62, 49-67.

[85] Potter, P.E. (1978) Petrology and Chemistry of Modern Big River Sands. The Journal of Geology, 86, 423-449. https://doi.org/10.1086/649711

[86] Hossain, I., Roy, K.K., Biswas, P.K., Alam, M., Moniruzzaman, Md. and Deeba, F. (2014) Geochemical Characteristics of Holocene Sediments from Chuadanga District, Bangladesh: Implications for Weathering, Climate, Redox Conditions, Provenance and Tectonic Setting. Chinese Journal of Geochemistry, 33, 336-350. https://doi.org/10.1007/s11631-014-0696-9

[87] Chen, M., Sun, M., Cai, K., Buslov, M.M., Zhao, G. and Rubanova, E.S. (2014) Geochemical Study of the Cambrian-Ordovician Metasedimentary Rocks from the Northern Altai-Mongolian Terrane, Northwestern Central Asian Orogenic Belt: Implications on the Provenance and Tectonic Setting. Journal of Asian Earth Sciences, 96, 69-83.

[88] Garcia, D., Coehlo, J. and Perrin, M. (1991) Fractionation between $\mathrm{TiO}_{2}$ and $\mathrm{Zr}$ as a Measure of Sorting within Shale and Sandstone Series (Northern Portugal). European Journal of Mineralogy, 3, 401-414. https://doi.org/10.1127/ejm/3/2/0401

[89] Armstrong-Altrin, J.S., Lee, Y.I., Kasper-Zubillaga, J.J. and Trejo-Ramírez, E. (2016) Mineralogy and Geochemistry of Sand along the Manzanillo and El Carrizal Beach Areas, Southern Mexico: Implications for Palaeoweathering, Provenance and Tectonic Setting. Geological Journal. https://doi.org/10.1002/gj.2792

[90] McLennan, S.M., Taylor, S.R., McCulloch, M.T. and Maynard, J.B. (1990) Geochemical and Nd-Sr Isotopic Composition of Deep Sea Turbidites: Crustal Evolution and Plate Tectonic Associations. Geochimimica et Cosmochimica Acta, 54, 2015-2050.

[91] Mongelli, G., Critelli, S., Perri, F., Sonnino, M. and Perrone, V. (2006) Sedimentary Recycling, Provenance and Paleoweathering from Chemistry and Mineralogy of Mesozoic Continental Redbeb Mudrocks, Peloritani Mountains, Southern Italy. Geochemical Journal, 40, 197-209.

[92] Perri, F., Critelli, S., Mongelli, G. and Cullers, R.L. (2011) Sedimentary Evolution of the Mesozoic Continental Redbeds Using Geochemical and Mineralogical Tools: The Case of Upper Triasic to Lowermost Jurassic Monte di Gioiosa Mudrocks (Sicily, Southern Italy). International Journal of Earth Sciences, 100, 1569-1587. https://doi.org/10.1007/s00531-010-0602-6

[93] Perri, F. (2014) Composition, Provenance and Source Weathering of Mesozoic Sandstones from Western-Central Mediterranean Alpine Chains. Journal of African Earth Sciences, 91, 32-43.

[94] Zou, S., Wu, C., Xu, D., Shan, Q., Zhang, X. and Hollings, P. (2016) Provenance and Depositional Setting of Lower Silurian Siliciclastic Rocks on Hainan Island, South China: Implications for a Passive Margin Environment of South China in Gondwana. Journal of Asian Earth Sciences, 123, 243-262.

[95] Jones, B. and Manning, D.A.C. (1994) Comparison of Geochemical Indices Used for the Interpretation of Palaeoredox Conditions in Ancient Mudstones. Chemical Geology, 111, 111-129.

[96] Rimmer, S.M. (2004) Geochemical Paleoredox Indicators in Devonian-Mississippian Black Shales, Central Appalachian Basin (USA). Chemical Geology, 206, 373-391.

[97] Hallberg, R.O. (1976) A Geochemical Method for Investigation of Palaeoredox Conditions in Sediments. Ambio Special Report, 4, 139-147. 
[98] Nath, B.N., Bau, M., Ramalingeswara Rao, B. and Rao, C.M. (1997) Trace and Rare Earth Elemental Variation in Arabian Sea Sediments through a Transect across the Oxygen Minimum Zone. Geochimimica et Cosmochimica Acta, 61, 2375-2388.

[99] Cullers, R.L. and Berendsen, P. (1998) The Provenance and Chemical Variation of Sandstones Associated with the Mid-Continent Rift System, USA. European Journal of Mineralogy, 10, 987-1002. https://doi.org/10.1127/ejm/10/5/0987

[100] Julleh Jalalur, M. and Suzuki, S. (2007) Geochemistry of Sandstones from the Miocene Surma Group, Bengal Basin, Bangladesh: Implications for Provenance, Tectonic Setting and Weathering. Geochemical Journal, 41, 415-428.

Submit or recommend next manuscript to SCIRP and we will provide best service for you:

Accepting pre-submission inquiries through Email, Facebook, LinkedIn, Twitter, etc. A wide selection of journals (inclusive of 9 subjects, more than 200 journals) Providing 24-hour high-quality service User-friendly online submission system Fair and swift peer-review system Efficient typesetting and proofreading procedure Display of the result of downloads and visits, as well as the number of cited articles Maximum dissemination of your research work

Submit your manuscript at: http://papersubmission.scirp.org/

Or contact ijg@scirp.org 